\title{
The Factorization for a Class of Hom-Coalgebras
}

\author{
Lihong Dong, ${ }^{1}$ Shuan Xue, ${ }^{1}$ and Xiaohui Zhang ${ }^{2}$ \\ ${ }^{1}$ College of Mathematics and Information Science, Henan Normal University, Xinxiang 453007, China \\ ${ }^{2}$ School of Mathematical Sciences, Qufu Normal University, Qufu 273165, China \\ Correspondence should be addressed to Lihong Dong; lihongdong2010@gmail.com
}

Received 15 May 2017; Revised 10 August 2017; Accepted 7 September 2017; Published 29 October 2017

Academic Editor: Yao-Zhong Zhang

Copyright (C) 2017 Lihong Dong et al. This is an open access article distributed under the Creative Commons Attribution License, which permits unrestricted use, distribution, and reproduction in any medium, provided the original work is properly cited.

Let $(H, \alpha)$ be a Hom-Hopf algebra and $(C, \beta)$ a Hom-coalgebra. In this paper, we first introduce the notions of Hom-crossed coproduct $C \rtimes_{\lambda} H$ and cleft coextension and then discuss the equivalence between them. Furthermore, we discuss the relation between cleft coextension and Hom-module coalgebra with the Hom-Hopf module structure and obtain a Hom-coalgebra factorization for Hom-module coalgebra with the Hom-Hopf module structure.

\section{Introduction}

The motivation to introduce Hom-type algebras comes for examples related to $q$-deformations of Witt and Virasoro algebras, which play an important role in physics, mainly in conformal field theory. Hom-structures (Lie algebras, algebras, coalgebras, Hopf algebras) have been intensively investigated recently. Makhlouf and Silvestrov $([1,2])$ generalized the associativity to twisted associativity and naturally proposed the notion of Hom-associative algebras and were first to introduce Hom-coalgebras, Hom-bialgebras, HomHopf algebras, and related objects.

The crossed products of algebras were independently introduced in [3, 4]. In [3], Blattner et al. showed the equivalence of crossed products and cleft extensions. In [5], Blattner and Montgomery gave several characterizations of crossed products. Lu and Wang [6] generalized the result in [3] to the case of Hom-Hopf algebras. The concept of crossed coproduct appeared as a dual version of the usual crossed product for Hopf algebras and it was studied in several papers; see [7-9]. In [8] the authors studied cleft coextension, a dual notion for that of cleft extension, and it was proved that a cleft coextension is isomorphic to a crossed coproduct. Naturally, what are the structure and the relation of crossed coproduct and cleft coextensions in the sense of Hom-structure?

In [10], Radford showed that a bialgebra with a projection had a factorization. In [11], Caenepeel et al. generalized Radford's result. It is natural to ask under what conditions a
Hom-coalgebra can be factorized into Hom-crossed coproduct. These two problems motivate us writing this paper.

This paper is organized as follows.

In Section 2, we recall some basic definitions and results, such as Hom-bialgebras, Hom-Hopf algebras, Hom(co)module, Hom-Hopf module, and Hom-module coalgebras. In Section 3, let $(H, \alpha)$ be a Hom-Hopf algebra and $(C, \beta)$ a Hom-coalgebra. We give the definition of Hom-Hopf algebra $H$ coacting weakly on Hom-coalgebra $C$ from the left, introduce the notion of Hom-crossed coproduct, and then discuss the necessary and sufficient conditions for $C \rtimes_{\lambda} H$ to be Hom-crossed coproduct (see Theorem 4). In Section 4, we introduce the definition of the cleft coextension and discuss the equivalence between the Hom-crossed coproducts and cleft coextensions (see Theorem 10). Furthermore, we discuss the relation between cleft coextension and Hom-module coalgebra with the Hom-Hopf module structure and obtain $(B, \cdot, \phi, \beta)$ which is a cleft coextension if and only if it is a right Hom-Hopf module (see Theorem 13). In Section 5, we obtain the Hom-coalgebra factorization for Hom-module coalgebra with the Hom-Hopf module structure (see Theorem 15).

\section{Preliminaries}

In this paper, all the vector spaces, tensor products, and homomorphisms are over a fixed field $k$. For a coalgebra $C$, we write $\Delta(c)=c_{1} \otimes c_{2}$ for any $c \in C$ (summation omitted). 
We now recall from [2,12-14] some definitions and results about the Hom-Hopf algebras, Hom-(co)modules, and so on.

2.1. Hom-Hopf Algebra. A Hom-algebra is a quadruple $\left(A, \mu, 1_{A}, \alpha\right)$ (abbr. $\left.(A, \alpha)\right)$, where $A$ is a linear space, $\mu$ : $A \otimes A \rightarrow A$ is a linear map, with notation $\mu\left(a \otimes a^{\prime}\right)=a a^{\prime}$, $1_{A} \in A$, and $\alpha \in A u t_{k}(A)$, such that, for any $a, a^{\prime}, a^{\prime \prime} \in A$,

$$
\begin{aligned}
\alpha\left(a a^{\prime}\right) & =\alpha(a) \alpha\left(a^{\prime}\right), \\
a 1_{A} & =1_{A} a=\alpha(a), \\
\alpha(a)\left(a^{\prime} a^{\prime \prime}\right) & =\left(a a^{\prime}\right) \alpha\left(a^{\prime \prime}\right), \\
\alpha\left(1_{A}\right) & =1_{A} .
\end{aligned}
$$

A linear map $f:\left(A, \mu_{A}, 1_{A}, \alpha_{A}\right) \rightarrow\left(B, \mu_{B}, 1_{B}, \alpha_{B}\right)$ is called a morphism of Hom-algebra if $\alpha_{B} f=f \alpha_{A}, f\left(1_{A}\right)=1_{B}$, and $f \mu_{A}=\mu_{B}(f \otimes f)$.

A Hom-coalgebra is a quadruple $(C, \Delta, \varepsilon, \beta)(\operatorname{abbr} .(C, \beta))$, where $C$ is a linear space, $\Delta: C \rightarrow C \otimes C, \varepsilon: C \rightarrow k$ are linear maps, and $\beta \in A u t_{k}(C)$, such that, for any $c \in C$,

$$
\begin{aligned}
\beta\left(c_{1}\right) \otimes \beta\left(c_{2}\right) & =\beta(c)_{1} \otimes \beta(c)_{2}, \\
\varepsilon\left(c_{1}\right) c_{2} & =c_{1} \varepsilon\left(c_{2}\right)=\beta(c), \\
\beta\left(c_{1}\right) \otimes c_{21} \otimes c_{22} & =c_{11} \otimes c_{12} \otimes \beta\left(c_{2}\right), \\
\varepsilon \beta & =\varepsilon .
\end{aligned}
$$

A linear map $f:\left(C, \Delta_{C}, \varepsilon_{C}, \beta_{C}\right) \rightarrow\left(D, \Delta_{D}, \varepsilon_{D}, \beta_{D}\right)$ is called a morphism of Hom-coalgebra if $\beta_{D} f=f \beta_{C}, \varepsilon_{D} f=$ $\varepsilon_{C}$, and $\Delta_{D} f=(f \otimes f) \Delta_{C}$.

A Hom-bialgebra is a sextuple $\left(H, \mu, 1_{H}, \Delta, \varepsilon, \gamma\right)$ (abbr. $(H, \gamma))$, where $\left(H, \mu, 1_{H}, \gamma\right)$ is a Hom-algebra and $(H, \Delta, \varepsilon, \gamma)$ is a Hom-coalgebra, such that $\Delta, \varepsilon$ are morphisms of Homalgebra; that is,

$$
\begin{aligned}
& \Delta\left(h h^{\prime}\right)=\Delta(h) \Delta\left(h^{\prime}\right), \\
& \Delta\left(1_{H}\right)=1_{H} \otimes 1_{H}, \\
& \varepsilon\left(h h^{\prime}\right)=\varepsilon(h) \varepsilon\left(h^{\prime}\right), \\
& \varepsilon\left(1_{H}\right)=1 .
\end{aligned}
$$

Furthermore, if there exists a linear map $S: H \rightarrow H$ such that

$$
\begin{aligned}
& S\left(h_{1}\right) h_{2}=h_{1} S\left(h_{2}\right)=\varepsilon(h) 1_{H}, \\
& S(\gamma(h))=\gamma(S(h)),
\end{aligned}
$$

then we call $\left(H, \mu, 1_{H}, \Delta, \varepsilon, S, \gamma\right)$ (abbr. $\left.(H, S, \gamma)\right)$ a Hom-Hopf algebra.

Let $(H, S, \gamma)$ be a Hom-Hopf algebra; for $S$ we have the following properties for any $h, g \in H$ :

$$
\begin{aligned}
S(h)_{1} \otimes S(h)_{2} & =S\left(h_{2}\right) \otimes S\left(h_{1}\right), \\
S(h g) & =S(g) S(h), \\
\varepsilon S & =\varepsilon .
\end{aligned}
$$

2.2. Hom-Hopf Module. Let $(A, \beta)$ be a Hom-algebra; a right $(A, \beta)$-Hom-module is a triple $(M, \cdot, \alpha)$, where $M$ is a linear space, $\cdot: M \otimes A \rightarrow M$ is a linear map, and $\alpha$ is an automorphism of $M$, such that, for any $a, a^{\prime} \in A$ and $m \in M$,

$$
\begin{aligned}
\alpha(m) \cdot\left(a a^{\prime}\right) & =(m \cdot a) \cdot \beta\left(a^{\prime}\right), \\
m \cdot 1_{A} & =\alpha(m), \\
\alpha(m \cdot a) & =\alpha(m) \cdot \beta(a) .
\end{aligned}
$$

Let $\left(M, \cdot_{M}, \alpha_{M}\right)$ and $\left(N,{ }_{N}, \alpha_{N}\right)$ be two right $(A, \beta)$-Hommodules. Then a linear morphism $f: M \rightarrow N$ is called a morphism of right $(A, \beta)$-Hom-modules if $f\left(m \cdot{ }_{M} a\right)=$ $f(m) \cdot{ }_{N} a$ and $\alpha_{M} f=f \alpha_{N}$.

Let $(C, \beta)$ be a Hom-coalgebra; a right $(C, \beta)$-Homcomodule is a triple $(M, \rho, \alpha)$, where $M$ is a linear space, $\rho: M \rightarrow M \otimes C$ is a linear map (write $\rho(m)=m_{(0)} \otimes m_{(1)}$ ), and $\alpha$ is an automorphism of $M$, such that, for any $m \in M$,

$$
\begin{aligned}
\alpha\left(m_{(0)}\right) \otimes m_{(1) 1} \otimes m_{(1) 2} & =m_{(0)(0)} \otimes m_{(0)(1)} \otimes \beta\left(m_{(1)}\right) \\
m_{(0)} \varepsilon\left(m_{(1)}\right) & =\alpha(m) \\
\alpha(m)_{(0)} \otimes \alpha(m)_{(1)} & =\alpha\left(m_{(0)}\right) \otimes \beta\left(m_{(0)}\right) .
\end{aligned}
$$

Let $\left(M, \rho_{M}, \alpha_{M}\right)$ and $\left(N, \rho_{N}, \alpha_{N}\right)$ be two right $(C, \beta)$ Hom-comodules. Then a linear morphism $f: M \rightarrow$ $N$ is called a morphism of right $(C, \beta)$-Hom-comodules if $f(m)_{(0)} \otimes f(m)_{(1)}=f\left(m_{(0)}\right) \otimes m_{(1)}$ and $\alpha_{M} f=f \alpha_{N}$.

Let $(H, \alpha)$ be a Hom-Hopf algebra; a right $H$-Hom-Hopf module is a quadruple $(M, \cdot, \rho, \beta)$, where $M$ is a right $H$ Hom-module and a right $H$-Hom-comodule, such that, for all $m \in M, h \in H$,

$$
\rho(m \cdot h)=m_{(0)} \cdot h_{1} \otimes m_{(1)} h_{2} .
$$

2.3. The Fundamental Theorem of Hom-Hopf Module. Let $(H$, $\alpha)$ be a Hom-Hopf algebra and $(B, \cdot, \rho, \beta)$ a right $H$-HomHopf module, and set $A=B^{c o H}=\{b \in B \mid \rho(b)=\beta(b) \otimes 1\}$, then

$$
\begin{aligned}
\varphi: A \otimes H & \longrightarrow B, \\
b \otimes h & \longmapsto b \cdot h
\end{aligned}
$$

is an isomorphism of right $H$-Hom-Hopf module.

2.4. Hom-Module Coalgebra. Recall from [15], let $(H, \beta)$ be a Hom-Hopf algebra and $(C, \alpha)$ a Hom-coalgebra, and if $(C, \cdot, \alpha)$ is a left $(H, \beta)$-Hom-module, for all $c \in C, h \in H$, the following conditions hold:

$$
\begin{aligned}
(h \cdot c)_{1} \otimes(h \cdot c)_{2} & =h_{1} \cdot c_{1} \otimes h_{2} \cdot c_{2}, \\
\varepsilon(h \cdot c) & =\varepsilon(h) \varepsilon(c),
\end{aligned}
$$

then $(C, \cdot, \alpha)$ is called an $(H, \beta)$-Hom-module coalgebra.

\section{Hom-Crossed Coproducts}

Let $(H, \alpha)$ be a Hom-Hopf algebra and $(C, \beta)$ a Homcoalgebra. In this section, we give the definition of $H$ coacting 
weakly on $C$ from the left and introduce Hom-crossed coproduct. Then we discuss the necessary and sufficient conditions for $C \rtimes_{\lambda} H$ to be Hom-crossed coproduct and get some properties about it.

Definition 1. Let $(H, \alpha)$ be a Hom-Hopf algebra and $(C, \beta)$ a Hom-coalgebra. We say that $H$ coacts weakly on $C$ from the left if there is a linear map $C \rightarrow H \otimes C, c \mapsto c_{-1} \otimes c_{0}$ such that for all $c \in C$ the following conditions hold:

$$
\begin{aligned}
& (\mathrm{W} 1) \alpha^{2}\left(c_{-1}\right) \otimes c_{01} \otimes c_{02}=c_{1-1} c_{2-1} \otimes c_{10} \otimes c_{20}, \\
& (\mathrm{~W} 2) \varepsilon\left(c_{0}\right) c_{-1}=\varepsilon(c) 1, \\
& (\mathrm{~W} 3) \varepsilon\left(c_{-1}\right) c_{0}=\beta(c) .
\end{aligned}
$$

Definition 2. Let $(H, \alpha)$ be a Hom-Hopf algebra and $(C, \beta)$ a Hom-coalgebra. Assume that $H$ coacts weakly on $C$ from the left. Let $\lambda \in \operatorname{Hom}(C, H \otimes H)$ be a linear map; write $\lambda(c)=$ $\lambda_{1}(c) \otimes \lambda_{2}(c)$. Define $C \rtimes_{\lambda} H$, whose underlying vector space is $C \otimes H$ with the comultiplication given by

$$
\begin{array}{r}
\Delta_{\lambda}(c \otimes h)=c_{1} \otimes\left(\alpha^{-4}\left(c_{21-1}\right) \alpha^{-3}\left(\lambda_{1}\left(c_{22}\right)\right)\right) \alpha^{-1}\left(h_{1}\right) \\
\otimes \beta^{-2}\left(c_{210}\right) \otimes \alpha^{-2}\left(\lambda_{2}\left(c_{22}\right)\right) \alpha^{-1}\left(h_{2}\right) .
\end{array}
$$

We say that $\left(C \rtimes_{\lambda} H, \beta \otimes \alpha\right)$ is a Hom-crossed coproduct if $\Delta_{\lambda}$ is coassociative and $\varepsilon \otimes \varepsilon$ is the counit for all $c \in C$ and $h \in H$.

Remark 3. If the cocycle $\lambda$ is convolution invertible, we will denote its convolution inverse by $\lambda^{-1}(c)=\lambda_{1}^{-1}(c) \otimes \lambda_{2}^{-1}(c)$.

Theorem 4. $\left(C \rtimes_{\lambda} H, \beta \otimes \alpha\right)$ is a Hom-crossed coproduct if and only if the following conditions hold.

(CU) Normal Cocycle Condition

$$
(i d \otimes \varepsilon) \lambda=(\varepsilon \otimes i d) \lambda=1_{H} \varepsilon
$$

\section{(C) Cocycle Condition}

$$
\begin{aligned}
& \lambda_{1}\left(c_{1}\right) \alpha^{-1}\left(\lambda_{1}\left(c_{2}\right)_{1}\right) \otimes \lambda_{2}\left(c_{1}\right) \alpha^{-1}\left(\lambda_{1}\left(c_{2}\right)_{2}\right) \\
& \otimes \alpha\left(\lambda_{2}\left(c_{2}\right)\right) \\
& =\alpha^{-1}\left(c_{1-1}\right) \lambda_{1}\left(c_{2}\right) \otimes \alpha^{-1}\left(\lambda_{1}\left(c_{10}\right)\right) \alpha^{-1}\left(\lambda_{2}\left(c_{2}\right)_{1}\right) \\
& \otimes \alpha^{-1}\left(\lambda_{2}\left(c_{10}\right)\right) \alpha^{-1}\left(\lambda_{2}\left(c_{2}\right)_{2}\right) .
\end{aligned}
$$

\section{(TC) Twisted Comodule Condition}

$$
\begin{aligned}
\lambda_{1}\left(c_{1}\right) \alpha^{-2}\left(c_{2-11}\right) \otimes \lambda_{2}\left(c_{1}\right) \alpha^{-2}\left(c_{2-12}\right) \otimes c_{20} \\
=\alpha^{-1}\left(c_{1-1}\right) \lambda_{1}\left(c_{2}\right) \otimes \alpha^{-2}\left(c_{10-1}\right) \lambda_{2}\left(c_{2}\right) \\
\otimes \beta^{-1}\left(c_{100}\right) .
\end{aligned}
$$

Proof. Directly computing, we can get that $\varepsilon \otimes \varepsilon$ is the counit of $\left(C \rtimes_{\lambda} H, \beta \otimes \alpha\right)$ if and only if (CU) holds.
Now, we prove that if $\left(C \rtimes_{\lambda} H, \beta \otimes \alpha\right)$ is a Hom-crossed coproduct then the conditions (C) and (TC) are satisfied. Because of coassociativity of $\left(C \rtimes_{\lambda} H, \beta \otimes \alpha\right)$, we can get

$$
\begin{aligned}
\left(\Delta_{\lambda} \otimes(\beta \otimes \alpha)\right) \Delta_{\lambda}(c \otimes h)=c_{11} & \\
& \otimes\left(\alpha^{-4}\left(c_{121-1}\right) \alpha^{-3}\left(\lambda_{1}\left(c_{122}\right)\right)\right) \\
\cdot & \left(\left(\alpha^{-5}\left(c_{21-11}\right) \alpha^{-4}\left(\lambda_{1}\left(c_{22}\right)\right)\right) \alpha^{-2}\left(h_{11}\right)\right) \\
& \otimes \beta^{-2}\left(c_{1210}\right) \otimes \alpha^{-2}\left(\lambda_{2}\left(c_{122}\right)\right) \\
\cdot & \left.\left(\left(\alpha^{-5}\left(c_{21-12}\right) \alpha^{-4}\left(\lambda_{1}\left(c_{22}\right)\right)_{2}\right)\right) \alpha^{-2}\left(h_{12}\right)\right) \\
& \otimes \beta^{-1}\left(c_{210}\right) \otimes \alpha^{-1}\left(\lambda_{2}\left(c_{22}\right)\right) h_{2}=\alpha\left(c_{1}\right) \\
& \otimes\left(\alpha^{-3}\left(c_{21-1}\right) \alpha^{-2}\left(\lambda_{1}\left(c_{22}\right)\right)\right) h_{1} \otimes \beta^{-2}\left(c_{2101}\right) \\
& \otimes\left(\alpha^{-6}\left(c_{21021-1}\right) \alpha^{-5}\left(\lambda_{1}\left(c_{21022}\right)\right)\right) \\
\cdot & \left(\alpha^{-3}\left(\lambda_{2}\left(c_{22}\right)_{1}\right) \alpha^{-2}\left(h_{21}\right)\right) \otimes \beta^{-4}\left(c_{210210}\right) \\
& \otimes \alpha^{-4}\left(\lambda_{2}\left(c_{21022}\right)\right)\left(\alpha^{-3}\left(\lambda_{2}\left(c_{22}\right)_{2}\right) \alpha^{-2}\left(h_{22}\right)\right) \\
& =\left((\beta \otimes \alpha) \otimes \Delta_{\lambda}\right) \Delta_{\lambda}(c \otimes h) .
\end{aligned}
$$

Taking $h=1_{H}$ in the above equality, then applying $(\varepsilon \otimes i d)^{2} \otimes$ $i d \otimes \varepsilon$ to both sides, and using (CU), we obtain (TC); and applying $(\varepsilon \otimes i d)^{3}$ to both sides, $(\mathrm{C})$ is obtained too.

Conversely, suppose (C) and (TC) hold, then

$$
\begin{aligned}
\left((\beta \otimes \alpha) \otimes \Delta_{\lambda}\right) \Delta_{\lambda}(c \otimes h) \stackrel{(\mathrm{W} 1)}{=} \alpha\left(c_{1}\right) \otimes\left(\left(\alpha^{-5}\left(c_{211-1}\right)\right.\right. \\
\left.\left.\cdot \alpha^{-7}\left(c_{2121-1} c_{2122-1}\right)\right) \alpha^{-2}\left(\lambda_{1}\left(c_{22}\right)\right)\right) h_{1} \\
\otimes \beta^{-2}\left(c_{2110}\right) \otimes\left(\alpha^{-6}\left(c_{21210-1}\right) \alpha^{-5}\left(\lambda_{1}\left(c_{21220}\right)\right)\right) \\
\cdot\left(\alpha^{-3}\left(\lambda_{2}\left(c_{22}\right)_{1}\right) \alpha^{-2}\left(h_{21}\right)\right) \otimes \beta^{-4}\left(c_{212100}\right) \\
\otimes \alpha^{-4}\left(\lambda_{2}\left(c_{21220}\right)\right)\left(\alpha^{-3}\left(\lambda_{2}\left(c_{22}\right)_{2}\right) \alpha^{-2}\left(h_{22}\right)\right) \\
=\alpha\left(c_{1}\right) \otimes\left(( \alpha ^ { - 5 } ( c _ { 2 1 1 - 1 } ) \alpha ^ { - 5 } ( c _ { 2 1 2 - 1 } ) ) \left(\alpha^{-5}\left(c_{221-1}\right)\right.\right. \\
\left.\left.\cdot \alpha^{-4}\left(\lambda_{1}\left(c_{222}\right)\right)\right)\right) h_{1} \otimes \beta^{-2}\left(c_{2110}\right) \otimes \alpha^{-4}\left(c_{2120-1}\right) \\
\cdot\left(\left(\alpha^{-5}\left(\lambda_{1}\left(c_{2210}\right)\right) \alpha^{-5}\left(\lambda_{2}\left(c_{222}\right)_{1}\right)\right) \alpha^{-2}\left(h_{21}\right)\right) \\
\otimes \beta^{-3}\left(c_{21200}\right) \otimes\left(\alpha^{-4}\left(\lambda_{2}\left(c_{2210}\right)\right) \alpha^{-4}\left(\lambda_{2}\left(c_{222}\right)_{2}\right)\right) \\
\cdot \alpha^{-1}\left(h_{22}\right) \stackrel{(\mathrm{C})}{=} \alpha\left(c_{1}\right) \otimes\left(\left(\alpha^{-5}\left(c_{211-1}\right) \alpha^{-5}\left(c_{212-1}\right)\right)\right. \\
\left.\cdot\left(\alpha^{-4}\left(\lambda_{1}\left(c_{221}\right)\right) \alpha^{-5}\left(\lambda_{1}\left(c_{222}\right)_{1}\right)\right)\right) h_{1} \otimes \beta^{-2}\left(c_{2110}\right) \\
\otimes \alpha^{-4}\left(c_{2120-1}\right)\left(\left(\alpha^{-4}\left(\lambda_{2}\left(c_{221}\right)\right) \alpha^{-5}\left(\lambda_{1}\left(c_{222}\right)_{2}\right)\right)\right. \\
\left.\cdot \alpha^{-2}\left(h_{21}\right)\right) \otimes \beta^{-3}\left(c_{21200}\right) \otimes \alpha^{-2}\left(\lambda_{2}\left(c_{222}\right)\right) \otimes\left(\alpha^{-3}\left(c_{21-1}\right)\right. \\
\left.\cdot \alpha^{-1}\right)
\end{aligned}
$$




$$
\begin{aligned}
& \left.\cdot\left(\left(\alpha^{-7}\left(c_{2211-1}\right) \alpha^{-6}\left(\lambda_{1}\left(c_{2212}\right)\right)\right) \alpha^{-5}\left(\lambda_{1}\left(c_{222}\right)_{1}\right)\right)\right) \\
& \cdot h_{1} \otimes \beta^{-1}\left(c_{210}\right) \otimes\left(\alpha^{-6}\left(c_{22110-1}\right) \alpha^{-4}\left(\lambda_{2}\left(c_{2212}\right)\right)\right) \\
& \cdot\left(\alpha^{-4}\left(\lambda_{1}\left(c_{222}\right)_{2}\right) \alpha^{-2}\left(h_{21}\right)\right) \otimes \beta^{-4}\left(c_{221100}\right) \\
& \otimes \alpha^{-2}\left(\lambda_{2}\left(c_{222}\right)\right) \alpha^{-1}\left(h_{22}\right) \stackrel{(\mathrm{TC})}{=} \alpha\left(c_{1}\right) \otimes\left(\alpha^{-3}\left(c_{21-1}\right)\right. \\
& \cdot\left(\left(\alpha^{-6}\left(\lambda_{1}\left(c_{2211}\right)\right) \alpha^{-8}\left(c_{2212-11}\right)\right)\right. \\
& \left.\left.\cdot \alpha^{-5}\left(\lambda_{1}\left(c_{222}\right)_{1}\right)\right)\right) h_{1} \otimes \beta^{-1}\left(c_{210}\right) \\
& \otimes\left(\alpha^{-4}\left(\lambda_{2}\left(c_{2211}\right)\right) \alpha^{-6}\left(c_{2212-12}\right)\right)\left(\alpha^{-4}\left(\lambda_{1}\left(c_{222}\right)_{2}\right)\right. \\
& \left.\cdot \alpha^{-2}\left(h_{21}\right)\right) \otimes \beta^{-3}\left(c_{22120}\right) \otimes \alpha^{-2}\left(\lambda_{2}\left(c_{222}\right)\right) \\
& \cdot \alpha^{-1}\left(h_{22}\right)=\alpha\left(c_{1}\right) \otimes\left(\alpha^{-3}\left(c_{21-1}\right) \alpha^{-4}\left(\lambda_{1}\left(c_{2211}\right)\right)\right) \\
& \left.\cdot\left(\left(\alpha^{-7}\left(c_{2212-11}\right) \alpha^{-5}\left(\lambda_{1}\left(c_{222}\right)\right)_{1}\right)\right) \alpha^{-2}\left(h_{11}\right)\right) \\
& \otimes \beta^{-1}\left(c_{210}\right) \otimes \alpha^{-3}\left(\lambda_{2}\left(c_{2211}\right)\right)\left(\left(\alpha^{-7}\left(c_{2212-12}\right)\right.\right. \\
& \otimes \alpha^{-2}\left(\lambda_{2}\left(c_{222}\right)\right) h_{2}=\left(c_{\lambda} \otimes\left(\beta \otimes \alpha_{222}\right)\right) \alpha_{\lambda}(c \otimes h)
\end{aligned}
$$

This completes the proof.

Example 5. (1) Consider the case when $\lambda$ is trivial, that is, $\lambda(c)=\varepsilon(c) 1_{H} \otimes 1_{H}$ for all $c \in C$. Then the Hom-crossed coproduct is reduced to Hom-smash coproduct.

Proof. If $\lambda(c)=\varepsilon(c) 1_{H} \otimes 1_{H}, \forall c \in C$, then $(i d \otimes \varepsilon) \lambda(c)=$ $\varepsilon(c)(i d \otimes \varepsilon)\left(1_{H} \otimes 1_{H}\right)=1_{H} \varepsilon(c)$. Similarly we can get $(\varepsilon \otimes i d) \lambda=$ $1_{H} \varepsilon$, so $(\mathrm{CU})$ is satisfied.

For condition (C), the left hand side is

$$
\begin{aligned}
& \lambda_{1}\left(c_{1}\right) \alpha^{-1}\left(\lambda_{1}\left(c_{2}\right)_{1}\right) \otimes \lambda_{2}\left(c_{1}\right) \alpha^{-1}\left(\lambda_{1}\left(c_{2}\right)_{2}\right) \\
& \otimes \alpha\left(\lambda_{2}\left(c_{2}\right)\right)=\varepsilon\left(c_{1}\right) \varepsilon\left(c_{2}\right) 1_{H} \otimes 1_{H} \otimes 1_{H} \\
& =\varepsilon(c) 1_{H} \otimes 1_{H} \otimes 1_{H},
\end{aligned}
$$

and the right hand side is

$$
\begin{aligned}
& \alpha^{-1}\left(c_{1-1}\right) \lambda_{1}\left(c_{2}\right) \otimes \alpha^{-1}\left(\lambda_{1}\left(c_{10}\right)\right) \alpha^{-1}\left(\lambda_{2}\left(c_{2}\right)_{1}\right) \\
& \otimes \alpha^{-1}\left(\lambda_{2}\left(c_{10}\right)\right) \alpha^{-1}\left(\lambda_{2}\left(c_{2}\right)_{2}\right) \\
& =\alpha^{-1}\left(c_{1-1}\right) \varepsilon\left(c_{2}\right) 1_{H} \otimes \alpha^{-1}\left(\lambda_{1}\left(c_{10}\right)\right) 1_{H} \\
& \otimes \alpha^{-1}\left(\lambda_{2}\left(c_{10}\right)\right) 1_{H}=\alpha^{-1}\left(c_{1-1}\right) \varepsilon\left(c_{2}\right) 1_{H} \\
& \otimes \varepsilon\left(c_{10}\right) 1_{H} \otimes 1_{H}=\varepsilon(c) 1_{H} \otimes 1_{H} \otimes 1_{H},
\end{aligned}
$$

so $(\mathrm{C})$ is satisfied.
For condition (TC), the left hand side is

$$
\begin{aligned}
\lambda_{1} & \left(c_{1}\right) \alpha^{-2}\left(c_{2-11}\right) \otimes \lambda_{2}\left(c_{1}\right) \alpha^{-2}\left(c_{2-12}\right) \otimes c_{20} \\
& =\varepsilon\left(c_{1}\right) \alpha^{-1}\left(c_{2-11}\right) \otimes \alpha^{-1}\left(c_{2-12}\right) \otimes c_{20} \\
& =c_{-11} \otimes c_{-12} \otimes \beta\left(c_{0}\right),
\end{aligned}
$$

the right hand side is

$$
\begin{aligned}
& \alpha^{-1}\left(c_{1-1}\right) \lambda_{1}\left(c_{2}\right) \otimes \alpha^{-2}\left(c_{10-1}\right) \lambda_{2}\left(c_{2}\right) \otimes \beta^{-1}\left(c_{100}\right) \\
& \quad=c_{1-1} \varepsilon\left(c_{2}\right) \otimes \alpha^{-1}\left(c_{10-1}\right) \otimes \beta^{-1}\left(c_{100}\right) \\
& \quad=\alpha\left(c_{-1}\right) \otimes c_{0-1} \otimes c_{00},
\end{aligned}
$$

and then the Hom-crossed coproduct is Hom-smash coproduct. In this case, $C$ is a left $H$-comodule coalgebra, and the condition (TC) is satisfied.

(2) Let $\mathrm{H}_{2}$ be 2-dimension Hopf group algebra with a basis $\{1, g\}$. Then $\left(H_{2}, i d\right)$ forms a Hom-Hopf algebra. Let $H_{4}$ be a vector space with a basis $\{e, c, x, y\}$. Define the Homcoalgebra structure on $H_{4}$ as follows.

The automorphism $\beta: H_{4} \rightarrow H_{4}$ is given by

$$
\begin{aligned}
& \beta(e)=e, \\
& \beta(c)=c, \\
& \beta(x)=-x, \\
& \beta(y)=-y ;
\end{aligned}
$$

the comultiplication and counit are given by

$$
\begin{aligned}
& \Delta(e)=e \otimes e, \\
& \Delta(c)=c \otimes c, \\
& \Delta(x)=-x \otimes c-e \otimes x, \\
& \Delta(y)=-y \otimes e-c \otimes y, \\
& \varepsilon(e)=1, \\
& \mathcal{\varepsilon}(c)=1, \\
& \varepsilon(x)=0, \\
& \varepsilon(y)=0 .
\end{aligned}
$$

It is easy to see that $\mathrm{H}_{4}$ is a Hom-coalgebra. by

Now consider the coaction $\rho^{H_{4}}: H_{4} \rightarrow H_{2} \otimes H_{4}$ defined

$$
\begin{gathered}
e_{-1} \otimes e_{0}:=1 \otimes e, \\
c_{-1} \otimes c_{0}:=1 \otimes c, \\
x_{-1} \otimes x_{0}:=-g \otimes x, \\
y_{-1} \otimes y_{0}:=-g \otimes y .
\end{gathered}
$$

Then after a direct computation, we get that $\mathrm{H}_{2}$ coacts weakly on $H_{4}$ from the left. Further, recall from (1) that if we define $\lambda: H_{4} \rightarrow H_{2} \otimes H_{2}$ by $\lambda(h)=\varepsilon(h) 1 \otimes 1$, then $\left(H_{4} \rtimes_{\lambda} H_{2}, \beta \otimes i d\right)$ is a Hom-crossed coproduct. 
Proof. We can prove that the condition (CU) and the condition (C) hold for any $c \in H_{4}$ the same as Example 5(1). Then we only prove that the condition (TC) holds. By the proof of Example 5(1), for $x \in H_{4}$, we can get that the left hand side of the condition (TC) is

$$
\begin{aligned}
& \lambda_{1}\left(x_{1}\right) x_{2-11} \otimes \lambda_{2}\left(x_{1}\right) x_{2-12} \otimes x_{20} \\
& \quad=x_{-11} \otimes x_{-12} \otimes \beta\left(x_{0}\right)=g \otimes x \otimes x
\end{aligned}
$$

and the right hand side of the condition (TC) is

$$
\begin{gathered}
x_{1-1} \lambda_{1}\left(x_{2}\right) \otimes x_{10-1} \lambda_{2}\left(x_{2}\right) \otimes \beta^{-1}\left(x_{100}\right) \\
=x_{-1} \otimes x_{0-1} \otimes x_{00}=g \otimes x \otimes x,
\end{gathered}
$$

so the condition (TC) holds for $x \in H_{4}$. Similarly, we can get the condition (TC) holds for $e, c, y \in H_{4}$.

(3) Let $(H, \Delta, \mu)$ be a Hopf algebra and $(C, \Delta)$ a coalgebra, and $H$ weakly coacts on $C$ from the left. Assume that $\alpha$ is a Hopf automorphism of $H$ and $\beta$ is a coalgebra isomorphism of $C$. Then we have Hom-Hopf algebra $\left(H_{\alpha}, \Delta_{\alpha}, \mu_{\alpha}, \alpha\right)$ and Hom-coalgebra $\left(C_{\beta}, \Delta_{\beta}, \beta\right)$ (see [2]). Furthermore assume that $\rho(\beta(c))=(\alpha \otimes \beta) \rho(c)$, and define the coaction $\rho(c)=$ $(\alpha \otimes \beta) \rho(c)$, then $H_{\alpha}$ weakly coacts on $C_{\beta}$ from the left. If $C \rtimes_{\lambda} H$ is a crossed coproduct and $\lambda \beta=(\alpha \otimes \alpha) \lambda$, then $C_{\beta} \rtimes_{\lambda} H_{\alpha}$ is a Hom-crossed coproduct.

Proof. If $C \rtimes_{\lambda} H$ is a crossed coproduct, then we can get

$$
\begin{aligned}
& (i d \otimes \varepsilon) \lambda=(\varepsilon \otimes i d) \lambda=1_{H} \varepsilon ; \\
& \lambda_{1}\left(c_{1}\right) \lambda_{1}\left(c_{2}\right)_{1} \otimes \lambda_{2}\left(c_{1}\right) \lambda_{1}\left(c_{2}\right)_{2} \otimes \lambda_{2}\left(c_{2}\right) \\
& \quad=c_{1-1} \lambda_{1}\left(c_{2}\right) \otimes \lambda_{1}\left(c_{10}\right) \lambda_{2}\left(c_{2}\right)_{1} \\
& \otimes \lambda_{2}\left(c_{10}\right) \lambda_{2}\left(c_{2}\right)_{2} ; \\
& \lambda_{1}\left(c_{1}\right) c_{2-11} \otimes \lambda_{2}\left(c_{1}\right) c_{2-12} \otimes c_{20} \\
& =c_{1-1} \lambda_{1}\left(c_{2}\right) \otimes c_{10-1} \lambda_{2}\left(c_{2}\right) \otimes c_{100} .
\end{aligned}
$$

From [2], we know that the multiplication and comultiplication of Hom-Hopf algebra $\left(H_{\alpha}, \Delta_{\alpha}, \mu_{\alpha}, \alpha\right)$ are, respectively, given by

$$
\begin{aligned}
\mu_{\alpha}(h \otimes g) & =\alpha(h) \alpha(g), \\
\Delta_{\alpha}(h) & =\alpha\left(h_{1}\right) \otimes \alpha\left(h_{2}\right),
\end{aligned}
$$

$$
\forall h, g \in H_{\alpha}
$$

and the comultiplication of Hom-coalgebra $\left(C_{\beta}, \Delta_{\beta}, \beta\right)$ is given by

$$
\Delta_{\beta}(c)=\beta\left(c_{1}\right) \otimes \beta\left(c_{2}\right), \quad \forall c \in C_{\beta} .
$$

First, we prove that $H_{\alpha}$ weakly coacts on $C_{\beta}$ from the left. We only prove that (W1) of Definition 1 holds; the other two are easy to get. For any $c \in C_{\beta}$,

$$
\begin{gathered}
\alpha^{2}\left(c_{-1}\right) \otimes c_{01} \otimes c_{02} \quad\left(\text { in } H_{\alpha} \otimes C_{\beta} \otimes C_{\beta}\right) \\
=\alpha^{3}\left(c_{-1}\right) \otimes \beta^{2}\left(c_{01}\right) \otimes \beta^{2}\left(c_{02}\right) \quad(\text { in } H \otimes C \otimes C) \\
=\alpha^{3}\left(c_{1-1} c_{2-1}\right) \otimes \beta^{2}\left(c_{10}\right) \otimes \beta^{2}\left(c_{20}\right) \quad(\text { in } H \otimes C \otimes C) \\
=c_{1-1} c_{2-1} \otimes c_{10} \otimes c_{20}, \quad\left(\text { in } H_{\alpha} \otimes C_{\beta} \otimes C_{\beta}\right),
\end{gathered}
$$

so $H_{\alpha}$ weakly coacts on $C_{\beta}$ from the left.

Then we prove that $C_{\beta} \rtimes_{\lambda} H_{\alpha}$ is a Hom-crossed coproduct. It is easy to see that the condition (CU) holds. For the condition (C),

$$
\begin{aligned}
& \lambda_{1}\left(c_{1}\right) \alpha^{-1}\left(\lambda_{1}\left(c_{2}\right)_{1}\right) \otimes \lambda_{2}\left(c_{1}\right) \alpha^{-1}\left(\lambda_{1}\left(c_{2}\right)_{2}\right) \\
& \quad \otimes \alpha\left(\lambda_{2}\left(c_{2}\right)\right) \quad\left(\text { in } H_{\alpha} \otimes H_{\alpha} \otimes H_{\alpha}\right) \\
& =\alpha^{2}\left(\lambda_{1}\left(c_{1}\right) \lambda_{1}\left(c_{2}\right)_{1}\right) \otimes \alpha^{2}\left(\lambda_{2}\left(c_{1}\right) \lambda_{1}\left(c_{2}\right)_{2}\right) \\
& \quad \otimes \alpha^{2}\left(\lambda_{2}\left(c_{2}\right)\right) \quad(\text { in } H \otimes H \otimes H) \\
& \stackrel{(27)}{=} \alpha^{2}\left(c_{1-1} \lambda_{1}\left(c_{2}\right)\right) \otimes \alpha^{2}\left(\lambda_{1}\left(c_{10}\right) \lambda_{2}\left(c_{2}\right)_{1}\right) \\
& \quad \otimes \alpha^{2}\left(\lambda_{2}\left(c_{10}\right) \lambda_{2}\left(c_{2}\right)_{2}\right) \quad(\text { in } H \otimes H \otimes H) \\
& =\alpha^{-1}\left(c_{1-1}\right) \lambda_{1}\left(c_{2}\right) \otimes \alpha^{-1}\left(\lambda_{1} \lambda_{1}\left(c_{10}\right)\right) \alpha^{-1}\left(\lambda_{2}\left(c_{2}\right)_{1}\right) \\
& \otimes \alpha^{-1}\left(\lambda_{2}\left(c_{10}\right)\right) \alpha^{-1}\left(\lambda_{2}\left(c_{2}\right)_{2}\right)
\end{aligned}
$$

$$
\text { (in } H_{\alpha} \otimes H_{\alpha} \otimes H_{\alpha} \text { ) }
$$

so the condition (CU) holds. Similarly we can prove that the condition (TC) holds by (28).

For a Hom-crossed coproduct, we can get the following properties, which are useful for the latter conclusions.

Lemma 6. Let $C \rtimes_{\lambda} H$ be a Hom-crossed coproduct with invertible cocycle $\lambda$. Then the following equalities hold for any $c \in C$ :

(i)

$$
\begin{aligned}
c_{-1} \otimes & \lambda_{1}\left(c_{0}\right) \otimes \lambda_{2}\left(c_{0}\right) \\
= & \left(\alpha^{-3}\left(\lambda_{1}\left(c_{11}\right)\right) \alpha^{-4}\left(\lambda_{1}\left(c_{12}\right)_{1}\right)\right) \alpha^{-1}\left(\lambda_{1}^{-1}\left(c_{2}\right)\right) \\
& \otimes\left(\alpha^{-3}\left(\lambda_{2}\left(c_{11}\right)\right) \alpha^{-4}\left(\lambda_{1}\left(c_{12}\right)_{2}\right)\right) \alpha^{-2}\left(\lambda_{2}^{-1}\left(c_{2}\right)_{1}\right) \\
& \otimes \alpha^{-2}\left(\lambda_{2}\left(c_{12}\right)\right) \alpha^{-2}\left(\lambda_{2}^{-1}\left(c_{2}\right)_{2}\right) ;
\end{aligned}
$$

(ii)

$$
\begin{aligned}
c_{-1} & \otimes \lambda_{1}^{-1}\left(c_{0}\right) \otimes \lambda_{2}^{-1}\left(c_{0}\right) \\
= & \alpha^{-1}\left(\lambda_{1}\left(c_{1}\right)\right)\left(\alpha^{-4}\left(\lambda_{1}^{-1}\left(c_{21}\right)_{1}\right) \alpha^{-3}\left(\lambda_{1}^{-1}\left(c_{22}\right)\right)\right) \\
& \otimes \alpha^{-2}\left(\lambda_{2}\left(c_{1}\right)_{1}\right)\left(\alpha^{-4}\left(\lambda_{1}^{-1}\left(c_{21}\right)_{2}\right) \alpha^{-3}\left(\lambda_{2}^{-1}\left(c_{22}\right)\right)\right) \\
& \otimes \alpha^{-2}\left(\lambda_{2}\left(c_{1}\right)_{2}\right) \alpha^{-2}\left(\lambda_{2}^{-1}\left(c_{21}\right)\right) .
\end{aligned}
$$


Proof. Applying $\alpha^{-3} \otimes \alpha^{-3} \otimes \alpha^{-3}$ to both sides of $(\mathrm{C})$ and then multiplying it (by convolution) to the right by the following map, we can get (i):

$$
c \longmapsto \alpha^{-1} \lambda_{1}^{-1}(c) \otimes \alpha^{-2}\left(\lambda_{2}^{-1}(c)_{1}\right) \otimes \alpha^{-2}\left(\lambda_{2}^{-1}(c)_{2}\right) .
$$

Let us denote by $F_{1}, F_{2}, F_{3}: C \rightarrow H \otimes H \otimes H$, where the map is defined by

$$
F_{1}(c)=c_{-1} \otimes \lambda_{1}\left(c_{0}\right) \otimes \lambda_{2}\left(c_{0}\right) .
$$

We get from (i) that a right convolution inverse for $F_{1}$ is the map $F_{2}$, which is given by

$$
\begin{aligned}
& F_{2}(c) \\
& =\alpha^{-1}\left(\lambda_{1}\left(c_{1}\right)\right)\left(\alpha^{-4}\left(\lambda_{1}^{-1}\left(c_{21}\right)_{1}\right) \alpha^{-3}\left(\lambda_{1}^{-1}\left(c_{22}\right)\right)\right) \\
& \quad \otimes \alpha^{-2}\left(\lambda_{2}\left(c_{1}\right)_{1}\right)\left(\alpha^{-4}\left(\lambda_{1}^{-1}\left(c_{21}\right)_{2}\right) \alpha^{-3}\left(\lambda_{2}^{-1}\left(c_{22}\right)\right)\right) \\
& \quad \otimes \alpha^{-2}\left(\lambda_{2}\left(c_{1}\right)_{2}\right) \alpha^{-2}\left(\lambda_{2}^{-1}\left(c_{21}\right)\right) .
\end{aligned}
$$

Let $F_{3}$ be given by $F_{3}(c)=c_{-1} \otimes \lambda_{1}^{-1}\left(c_{0}\right) \otimes \lambda_{2}^{-1}\left(c_{0}\right)$. Since $\left(F_{3} *\right.$ $\left.F_{1}\right)(c)=\varepsilon(c) 1 \otimes 1 \otimes 1$. We get that $F_{3}$ is a left convolution inverse of $F_{1}$, so $F_{3}=F_{2}$. Therefore, we prove that (ii) holds.

Remark 7. Note that if $\left(C \rtimes_{\lambda} H, \beta \otimes \alpha\right)$ is a Hom-crossed coproduct, then the map $\pi: C \rtimes_{\lambda} H \rightarrow C$, defined by $\pi(c \otimes h)=\varepsilon(h) \beta^{-1}(c)$, is a Hom-coalgebra map, and we will also define the map $\theta: C \rtimes_{\lambda} H \rightarrow H, \theta(c \otimes h)=\varepsilon(c) \alpha^{-1}(h)$.

The following result is the generalization of Proposition 2.1 in [8].

Proposition 8. Let $B=C \rtimes_{\lambda} H$ be a Hom-crossed coproduct. Then $\theta$ is convolution invertible in $\operatorname{Hom}(B, H)$ if and only if $\lambda$ is convolution invertible in $\operatorname{Hom}(C, H \otimes H)$.

Proof. Assume first that $\lambda$ is convolution invertible; for any $c \rtimes h \in B$, define $\mu: B \rightarrow H$, by

$$
\mu(c \rtimes h)=S\left(\alpha^{-3}\left(\lambda_{1}^{-1}(c)\right) \alpha^{-3}(h)\right) \alpha^{-2}\left(\lambda_{2}^{-1}(c)\right) .
$$

Now we show that $\mu$ is a convolution inverse of $\theta$.

$$
\begin{aligned}
(\mu & * \theta)(c \rtimes h)=\mu\left(c_{1} \otimes\left(\alpha^{-4}\left(c_{21-1}\right) \alpha^{-3}\left(\lambda_{1}\left(c_{22}\right)\right)\right)\right. \\
\cdot & \left.\alpha^{-1}\left(h_{1}\right)\right) \theta\left(\beta^{-2}\left(c_{210}\right) \otimes \alpha^{-2}\left(\lambda_{2}\left(c_{22}\right)\right) \alpha^{-1}\left(h_{2}\right)\right) \\
& =S\left(\alpha^{-3}\left(\lambda_{1}^{-1}\left(c_{1}\right)\right)\right. \\
& \left.\cdot \alpha^{-3}\left(\left(\alpha^{-4}\left(c_{21-1}\right) \alpha^{-3}\left(\lambda_{1}\left(c_{22}\right)\right)\right) \alpha^{-1}\left(h_{1}\right)\right)\right) \\
& \cdot \alpha^{-2}\left(\lambda_{2}^{-1}\left(c_{1}\right)\right) \varepsilon\left(c_{210}\right)\left(\alpha^{-3}\left(\lambda_{2}\left(c_{22}\right)\right) \alpha^{-2}\left(h_{2}\right)\right) \\
& =S\left(\alpha^{-3}\left(\lambda_{1}^{-1}\left(c_{1}\right)\right)\left(\alpha^{-4}\left(\lambda_{1}\left(c_{2}\right)\right) \alpha^{-4}\left(h_{1}\right)\right)\right) \\
& \cdot \alpha^{-2}\left(\lambda_{2}^{-1}\left(c_{1}\right)\right)\left(\alpha^{-2}\left(\lambda_{2}\left(c_{2}\right)\right) \alpha^{-2}\left(h_{2}\right)\right) \\
& =S\left(\alpha^{-3}\left(\lambda_{1}^{-1}\left(c_{1}\right) \lambda_{1}\left(c_{2}\right)\right) \alpha^{-2}\left(h_{1}\right)\right) \\
& \cdot\left(\alpha^{-3}\left(\lambda_{2}^{-1}\left(c_{1}\right) \lambda_{2}\left(c_{2}\right)\right) \alpha^{-2}\left(h_{2}\right)\right)=\varepsilon(c) \varepsilon(h) .
\end{aligned}
$$

So $\mu$ is a left inverse for $\theta$; in order to show that $\mu$ is a right inverse we compute as follows:

$$
\begin{aligned}
& (\theta * \mu)(c \rtimes h)=\theta\left(c_{1} \otimes\left(\alpha^{-4}\left(c_{21-1}\right) \alpha^{-3}\left(\lambda_{1}\left(c_{22}\right)\right)\right)\right. \\
& \left.\cdot \alpha^{-1}\left(h_{1}\right)\right) \mu\left(\beta^{-2}\left(c_{210}\right) \otimes \alpha^{-2}\left(\lambda_{2}\left(c_{22}\right)\right) \alpha^{-1}\left(h_{2}\right)\right) \\
& =\varepsilon\left(c_{1}\right)\left(\alpha^{-5}\left(c_{21-1}\right) \alpha^{-4}\left(\lambda_{1}\left(c_{22}\right)\right)\right) \alpha^{-2}\left(h_{1}\right) \\
& \cdot\left(S\left(\alpha^{-5}\left(\lambda_{1}^{-1}\left(c_{210}\right)\right)\left(\alpha^{-5}\left(\lambda_{2}\left(c_{22}\right)\right) \alpha^{-4}\left(h_{2}\right)\right)\right)\right. \\
& \left.\cdot \alpha^{-4}\left(\lambda_{2}^{-1}\left(c_{210}\right)\right)\right)=\left(\alpha^{-4}\left(c_{1-1}\right) \alpha^{-3}\left(\lambda_{1}\left(c_{2}\right)\right)\right) \\
& \cdot \alpha^{-2}\left(h_{1}\right)\left(S \left(\left(\alpha^{-5}\left(\lambda_{1}^{-1}\left(c_{10}\right)\right) \alpha^{-4}\left(\lambda_{2}\left(c_{2}\right)\right)\right)\right.\right. \\
& \left.\left.\cdot \alpha^{-3}\left(h_{2}\right)\right) \alpha^{-3}\left(\lambda_{2}^{-1}\left(c_{10}\right)\right)\right)=\left(\alpha^{-3}\left(c_{1-1}\right)\right. \\
& \left.\cdot \alpha^{-2}\left(\lambda_{1}\left(c_{2}\right)\right)\right)\left(\left(\alpha^{-3}\left(h_{1}\right) S\left(\alpha^{-3}\left(h_{2}\right)\right)\right)\right. \\
& \cdot\left(S\left(\alpha^{-5}\left(\lambda_{1}^{-1}\left(c_{10}\right)\right) \alpha^{-4}\left(\lambda_{2}\left(c_{2}\right)\right)\right)\right. \\
& \left.\left.\cdot \alpha^{-4}\left(\lambda_{2}^{-1}\left(c_{10}\right)\right)\right)\right)=\left(\alpha^{-3}\left(c_{1-1}\right) \alpha^{-2}\left(\lambda_{1}\left(c_{2}\right)\right)\right) \\
& \cdot \varepsilon(h)\left(S\left(\alpha^{-4}\left(\lambda_{1}^{-1}\left(c_{10}\right)\right) \alpha^{-3}\left(\lambda_{2}\left(c_{2}\right)\right)\right)\right. \\
& \left.\cdot \alpha^{-3}\left(\lambda_{2}^{-1}\left(c_{10}\right)\right)\right) \stackrel{(34)}{=}\left(\left(\alpha^{-4}\left(\lambda_{1}\left(c_{11}\right)\right)\right.\right. \\
& \left.\cdot\left(\alpha^{-7}\left(\lambda_{1}^{-1}\left(c_{121}\right)_{1}\right) \alpha^{-6}\left(\lambda_{1}^{-1}\left(c_{122}\right)\right)\right)\right) \\
& \left.\cdot \alpha^{-2}\left(\lambda_{1}\left(c_{2}\right)\right)\right) \varepsilon(h)\left(S \left(\left(\alpha^{-6}\left(\lambda_{2}\left(c_{11}\right)_{1}\right)\right.\right.\right. \\
& \left.\cdot\left(\alpha^{-8}\left(\lambda_{1}^{-1}\left(c_{121}\right)_{2}\right) \alpha^{-7}\left(\lambda_{2}^{-1}\left(c_{122}\right)\right)\right)\right) \\
& \left.\left.\cdot \alpha^{-3}\left(\lambda_{2}\left(c_{2}\right)\right)\right)\left(\alpha^{-5}\left(\lambda_{2}\left(c_{11}\right)_{2}\right) \alpha^{-5}\left(\lambda_{2}^{-1}\left(c_{121}\right)\right)\right)\right) \\
& =\left(\alpha^{-3}\left(\lambda_{1}\left(c_{11}\right)\right)\left(\alpha^{-5}\left(\lambda_{1}^{-1}\left(c_{12}\right)_{1}\right) \varepsilon\left(c_{2}\right) 1\right)\right) \varepsilon(h) \\
& \cdot\left(S\left(\alpha^{-5}\left(\lambda_{2}\left(c_{11}\right)_{1}\right)\left(\alpha^{-6}\left(\lambda_{1}^{-1}\left(c_{12}\right)_{2}\right) 1\right)\right)\right. \\
& \left.\cdot\left(\alpha^{-5}\left(\lambda_{2}\left(c_{11}\right)_{2}\right) \alpha^{-4}\left(\lambda_{2}^{-1}\left(c_{12}\right)\right)\right)\right) \\
& =\left(\alpha^{-2}\left(\lambda_{1}\left(c_{1}\right)\right) \alpha^{-3}\left(\lambda_{1}^{-1}\left(c_{2}\right)_{1}\right)\right) \varepsilon(h) \\
& \cdot\left(S\left(\alpha^{-4}\left(\lambda_{2}\left(c_{1}\right)_{1}\right) \alpha^{-4}\left(\lambda_{1}^{-1}\left(c_{2}\right)_{2}\right)\right)\right. \\
& \left.\cdot\left(\alpha^{-4}\left(\lambda_{2}\left(c_{1}\right)_{2}\right) \alpha^{-3}\left(\lambda_{2}^{-1}\left(c_{2}\right)\right)\right)\right)=\varepsilon(c) \varepsilon(h) .
\end{aligned}
$$

So $\theta$ is convolution invertible.

Next we prove that if $\theta$ is convolution invertible, then $\lambda$ is also convolution invertible. Recalling

$$
\begin{aligned}
& \pi: B \longrightarrow C, \\
& c \otimes h \longmapsto \varepsilon(h) \beta^{-1}(c),
\end{aligned}
$$

we prove that $\lambda \pi$ is convolution invertible.

Firstly, we prove that

$$
\begin{aligned}
(\lambda \pi)(b)= & \alpha^{-3}\left(\theta\left(b_{11}\right)\right) \alpha^{-3}\left(\theta^{-1}\left(b_{2}\right)_{1}\right) \\
& \otimes \alpha^{-3}\left(\theta\left(b_{12}\right)\right) \alpha^{-3}\left(\theta^{-1}\left(b_{2}\right)_{2}\right) .
\end{aligned}
$$


Observe that if $b=c \otimes h$, then the equality $\theta\left(b_{1}\right) \theta^{-1}\left(b_{2}\right)=$ $\varepsilon(b) 1$ becomes

$$
\begin{aligned}
& \left(\left(\alpha^{-4}\left(c_{1-1}\right) \alpha^{-3}\left(\lambda_{1}\left(c_{2}\right)\right)\right) \alpha^{-2}\left(h_{1}\right)\right) \\
& \quad \cdot \theta^{-1}\left(\beta^{-1}\left(c_{10}\right) \otimes \alpha^{-1}\left(\lambda_{2}\left(c_{2}\right)\right) \alpha^{-1}\left(h_{2}\right)\right)=\varepsilon(c) \\
& \quad \cdot \varepsilon(h) 1 .
\end{aligned}
$$

By applying $\Delta_{H}$ we get

$$
\begin{aligned}
& \left(\left(\alpha^{-4}\left(c_{1-1}\right)_{1} \alpha^{-3}\left(\lambda_{1}\left(c_{2}\right)\right)_{1}\right) \alpha^{-2}\left(h_{1}\right)_{1}\right) \\
& \cdot \theta^{-1}\left(\beta^{-1}\left(c_{10}\right) \otimes \alpha^{-1}\left(\lambda_{2}\left(c_{2}\right)\right) \alpha^{-1}\left(h_{2}\right)\right)_{1} \\
& \otimes\left(\left(\alpha^{-4}\left(c_{1-1}\right)_{2} \alpha^{-3}\left(\lambda_{1}\left(c_{2}\right)\right)_{2}\right) \alpha^{-2}\left(h_{1}\right)_{2}\right)
\end{aligned}
$$

$\cdot \theta^{-1}\left(\beta^{-1}\left(c_{10}\right) \otimes \alpha^{-1}\left(\lambda_{2}\left(c_{2}\right)\right) \alpha^{-1}\left(h_{2}\right)\right)_{2}=\varepsilon(c)$

$\cdot \varepsilon(h) 1 \otimes 1$.

At the same time, we have

$$
\begin{aligned}
& b_{11} \otimes b_{12} \otimes b_{2}=c_{11} \otimes\left(\alpha^{-4}\left(c_{121-1}\right) \alpha^{-3}\left(\lambda_{1}\left(c_{122}\right)\right)\right) \\
& \cdot\left(\left(\alpha^{-5}\left(c_{21-11}\right) \alpha^{-4}\left(\lambda_{1}\left(c_{22}\right)_{1}\right)\right) \alpha^{-2}\left(h_{11}\right)\right) \\
& \otimes \beta^{-2}\left(c_{1210}\right) \otimes \alpha^{-2}\left(\lambda_{2}\left(c_{122}\right)\right) \\
& \cdot\left(\left(\alpha^{-5}\left(c_{21-12}\right) \alpha^{-4}\left(\lambda_{1}\left(c_{22}\right)_{2}\right)\right) \alpha^{-2}\left(h_{12}\right)\right) \\
& \otimes \beta^{-2}\left(c_{210}\right) \otimes \alpha^{-2}\left(\lambda_{2}\left(c_{22}\right)\right) \alpha^{-1}\left(h_{2}\right) .
\end{aligned}
$$

So we get

$$
\begin{aligned}
& \alpha^{-3}\left(\theta\left(b_{11}\right)\right) \alpha^{-3}\left(\theta^{-1}\left(b_{2}\right)_{1}\right) \otimes \alpha^{-3}\left(\theta\left(b_{12}\right)\right) \alpha^{-3}\left(\theta^{-1}\left(b_{2}\right)_{2}\right) \\
& =\alpha^{-3}\left(\lambda_{1}\left(c_{1}\right)\right)\left(\left(\left(\alpha^{-9}\left(c_{21-11}\right) \alpha^{-8}\left(\lambda_{1}\left(c_{22}\right)_{1}\right)\right) \alpha^{-6}\left(h_{11}\right)\right)\right) \theta^{-1}\left(\beta^{-6}\left(c_{210}\right) \otimes \alpha^{-6}\left(\lambda_{2}\left(c_{22}\right)\right) \alpha^{-5}\left(h_{2}\right)\right)_{1} \\
& \otimes \alpha^{-3}\left(\lambda_{2}\left(c_{1}\right)\right)\left(\left(\left(\alpha^{-9}\left(c_{21-12}\right) \alpha^{-8}\left(\lambda_{1}\left(c_{22}\right)_{2}\right)\right) \alpha^{-6}\left(h_{12}\right)\right)\right) \theta^{-1}\left(\beta^{-6}\left(c_{210}\right) \otimes \alpha^{-6}\left(\lambda_{2}\left(c_{22}\right)\right) \alpha^{-5}\left(h_{2}\right)\right)_{2}
\end{aligned}
$$

Secondly, let $\omega: B \rightarrow H \otimes H$, which is given by

$$
\begin{aligned}
\omega(b)= & \alpha^{-4}\left(\theta\left(b_{11}\right)_{1}\right) \alpha^{-2}\left(\theta^{-1}\left(b_{2}\right)\right) \\
& \otimes \alpha^{-4}\left(\theta\left(b_{11}\right)_{2}\right) \alpha^{-3}\left(\theta^{-1}\left(b_{12}\right)\right),
\end{aligned}
$$

and we prove that $\omega$ is the convolution inverse of $\lambda \pi$.

On the one hand,

$$
\begin{aligned}
& (\lambda \pi * \omega)(b)=(\lambda \pi)\left(b_{1}\right) \omega\left(b_{2}\right)=\left(\alpha^{-3}\left(\theta\left(b_{111}\right)\right)\right. \\
& \left.\cdot \alpha^{-3}\left(\theta^{-1}\left(b_{12}\right)_{1}\right)\right)\left(\alpha^{-4}\left(\theta\left(b_{211}\right)_{1}\right) \alpha^{-2}\left(\theta^{-1}\left(b_{22}\right)\right)\right) \\
& \otimes\left(\alpha^{-3}\left(\theta\left(b_{112}\right)\right) \alpha^{-3}\left(\theta^{-1}\left(b_{12}\right)_{2}\right)\right)\left(\alpha^{-4}\left(\theta\left(b_{211}\right)_{2}\right)\right. \\
& \left.\cdot \alpha^{-3}\left(\theta^{-1}\left(b_{212}\right)\right)\right)=\alpha^{-1}\left(\theta\left(b_{11}\right)\right) \\
& \cdot\left(\left(\alpha^{-5}\left(\theta^{-1}\left(b_{211}\right)_{1}\right) \alpha^{-5}\left(\theta\left(b_{212}\right)_{1}\right)\right)\right. \\
& \left.\cdot \alpha^{-3}\left(\theta^{-1}\left(b_{222}\right)\right)\right) \otimes \alpha^{-1}\left(\theta\left(b_{12}\right)\right) \\
& \cdot\left(\left(\alpha^{-5}\left(\theta^{-1}\left(b_{211}\right)_{2}\right) \alpha^{-5}\left(\theta\left(b_{212}\right)_{2}\right)\right)\right. \\
& \left.\cdot \alpha^{-3}\left(\theta^{-1}\left(b_{221}\right)\right)\right)=\alpha^{-1}\left(\theta\left(b_{11}\right)\right) \alpha^{-2}\left(\theta^{-1}\left(b_{222}\right)\right) \\
& \cdot \varepsilon\left(b_{21}\right) \otimes \alpha^{-1}\left(\theta\left(b_{12}\right)\right) \alpha^{-2}\left(\theta^{-1}\left(b_{221}\right)\right)=\theta\left(b_{1}\right) \\
& \cdot \alpha^{-1}\left(\theta^{-1}\left(b_{22}\right)\right) \otimes \alpha^{-2}\left(\theta\left(b_{211}\right)\right) \alpha^{-2}\left(\theta^{-1}\left(b_{222}\right)\right)
\end{aligned}
$$

$$
\begin{aligned}
& =\theta\left(b_{1}\right) \alpha^{-1}\left(\theta^{-1}\left(b_{22}\right)\right) \otimes 1 \varepsilon\left(b_{21}\right)=\theta\left(b_{1}\right) \theta^{-1}\left(b_{2}\right) \\
& \otimes 1=1 \otimes 1 \varepsilon(b) .
\end{aligned}
$$

So $\omega$ is a left convolution inverse for $\lambda \pi$. On the other hand,

$$
\begin{aligned}
& (\omega * \lambda \pi)(b)=\omega\left(b_{1}\right) \lambda \pi\left(b_{2}\right)=\left(\alpha^{-4}\left(\theta\left(b_{111}\right)_{1}\right)\right. \\
& \left.\cdot \alpha^{-2}\left(\theta^{-1}\left(b_{12}\right)\right)\right)\left(\alpha^{-3}\left(\theta\left(b_{211}\right)\right) \alpha^{-3}\left(\theta^{-1}\left(b_{22}\right)_{1}\right)\right) \\
& \otimes\left(\alpha^{-4}\left(\theta\left(b_{111}\right)_{2}\right) \alpha^{-3}\left(\theta^{-1}\left(b_{112}\right)\right)\right)\left(\alpha^{-3}\left(\theta\left(b_{212}\right)\right)\right. \\
& \left.\cdot \alpha^{-3}\left(\theta^{-1}\left(b_{22}\right)_{2}\right)\right)=\alpha^{-2}\left(\theta\left(b_{11}\right)_{1}\right) \\
& \cdot\left(\left(\alpha^{-4}\left(\theta^{-1}\left(b_{211}\right)\right) \alpha^{-4}\left(\theta\left(b_{212}\right)\right)\right) \alpha^{-4}\left(\theta^{-1}\left(b_{222}\right)_{1}\right)\right) \\
& \otimes \alpha^{-2}\left(\theta\left(b_{11}\right)_{2}\right)\left(\left(\alpha^{-3}\left(\theta^{-1}\left(b_{12}\right)\right) \alpha^{-4}\left(\theta\left(b_{221}\right)\right)\right)\right. \\
& \left.\cdot \alpha^{-4}\left(\theta^{-1}\left(b_{222}\right)_{2}\right)\right)=\alpha^{-2}\left(\theta\left(b_{11}\right)_{1}\right) \\
& \cdot \alpha^{-3}\left(\theta^{-1}\left(b_{222}\right)_{1}\right) \varepsilon\left(b_{21}\right) \otimes \alpha^{-2}\left(\theta\left(b_{11}\right)_{2}\right) \\
& \cdot\left(\left(\alpha^{-3}\left(\theta^{-1}\left(b_{12}\right)\right) \alpha^{-4}\left(\theta\left(b_{221}\right)\right)\right) \alpha^{-4}\left(\theta^{-1}\left(b_{222}\right)_{2}\right)\right) \\
& =\alpha^{-2}\left(\theta\left(b_{11}\right)_{1}\right) \alpha^{-2}\left(\theta^{-1}\left(b_{22}\right)_{1}\right) \otimes \alpha^{-2}\left(\theta\left(b_{11}\right)_{2}\right) \\
& \cdot\left(\left(\alpha^{-3}\left(\theta^{-1}\left(b_{12}\right)\right) \alpha^{-3}\left(\theta\left(b_{21}\right)\right)\right) \alpha^{-3}\left(\theta^{-1}\left(b_{22}\right)_{2}\right)\right) \\
& =\alpha^{-1}\left(\theta\left(b_{1}\right)_{1}\right) \alpha^{-2}\left(\theta^{-1}\left(b_{22}\right)_{1}\right) \otimes \alpha^{-1}\left(\theta\left(b_{1}\right)_{2}\right)
\end{aligned}
$$




$$
\begin{aligned}
& \cdot\left(\left(\alpha^{-4}\left(\theta^{-1}\left(b_{211}\right)\right) \alpha^{-4}\left(\theta\left(b_{212}\right)\right)\right) \alpha^{-3}\left(\theta^{-1}\left(b_{22}\right)_{2}\right)\right) \\
& =\alpha^{-1}\left(\theta\left(b_{1}\right)_{1}\right) \alpha^{-2}\left(\theta^{-1}\left(b_{22}\right)_{1}\right) \otimes \alpha^{-1}\left(\theta\left(b_{1}\right)_{2}\right) \\
& \cdot \alpha^{-2}\left(\theta^{-1}\left(b_{22}\right)_{2}\right) \varepsilon\left(b_{21}\right)=\alpha^{-1}\left(\theta\left(b_{1}\right)_{1}\right) \\
& \cdot \alpha^{-1}\left(\theta^{-1}\left(b_{2}\right)_{1}\right) \otimes \alpha^{-1}\left(\theta\left(b_{1}\right)_{2}\right) \alpha^{-1}\left(\theta^{-1}\left(b_{2}\right)_{2}\right) \\
& =1 \otimes 1 \varepsilon(d) .
\end{aligned}
$$

Thirdly, let $j: C \rightarrow B, j(c)=c \otimes 1$, then we have

$$
\begin{aligned}
(\pi & \otimes i d) \Delta(j(c))=(\pi \otimes i d) \Delta(\beta(c) \otimes 1) \\
= & \pi\left(\beta\left(c_{1}\right) \otimes \alpha^{-2}\left(c_{21-1}\right) \alpha^{-1}\left(\lambda_{1}\left(c_{22}\right)\right)\right) \\
& \otimes \beta^{-1}\left(c_{210}\right) \otimes \lambda_{2}\left(c_{22}\right) \\
= & c_{1} \varepsilon\left(c_{21-1}\right) \varepsilon\left(\lambda_{1}\left(c_{22}\right)\right) \otimes \beta^{-1}\left(c_{210}\right) \otimes \lambda_{2}\left(c_{22}\right) \\
= & c_{1} \otimes c_{21} \otimes \varepsilon\left(c_{22}\right) 1=c_{1} \otimes \beta\left(c_{2}\right) \otimes 1 \\
= & c_{1} \otimes j\left(c_{2}\right) .
\end{aligned}
$$

Since $(\lambda \pi) * \omega=1$, we obtain that

$$
\begin{aligned}
(\lambda \pi * \omega)(j(c)) & =(\lambda \pi)\left(j(c)_{1}\right) \omega\left(j(c)_{2}\right) \\
& =\lambda\left(c_{1}\right)(\omega j)\left(c_{2}\right)=\varepsilon(c) 1 .
\end{aligned}
$$

So $\lambda$ has a right convolution inverse $\omega j$ in $\operatorname{Hom}(C, H \otimes H)$. On the other hand,

$$
\begin{aligned}
(\lambda \pi * \omega j) \pi(b) & =\lambda\left(\pi\left(b_{1}\right)\right)(\omega j)\left(\pi\left(b_{2}\right)\right) \\
& =\lambda\left(\pi(b)_{1}\right)(\omega j)\left(\pi(b)_{2}\right) \\
& =\varepsilon(\pi(b)) 1=\varepsilon(b) 1 .
\end{aligned}
$$

It shows that $\omega j \pi$ is a right convolution inverse for $\lambda \pi$ in $\operatorname{Hom}(B, H \otimes H)$; thus $\omega j \pi=\omega$.

Finally, we denote that $\lambda^{\prime}=\omega j$, because we have that $(\lambda \pi) *\left(\lambda^{\prime} \pi\right)=\left(\lambda^{\prime} \pi\right) *(\lambda \pi)=1$ in $\operatorname{Hom}(B, H \otimes H)$ and $\pi$ is a surjective Hom-coalgebra map, and we obtain $\lambda * \lambda^{\prime}=$ $\lambda^{\prime} * \lambda=1$ in $\operatorname{Hom}(C, H \otimes H)$. So $\lambda^{\prime}$ is the convolution inverse of $\lambda$.

\section{Two Equivalent Characterizations of Cleft Coextensions}

In this section, we introduce the notion of the cleft coextension and then discuss two equivalent characterizations of cleft coextensions.

Let $(H, \alpha)$ be a Hom-Hopf algebra and $(B, \beta)$ a right $H$ Hom-module coalgebra. Let $H^{+}$denote the augmentation Hom-ideal ker $\varepsilon$ which is a Hom-Hopf ideal, then $B H^{+}$is a Hom-coideal of $B$, and $B / B H^{+}$is a Hom-coalgebra with a trivial right $H$-Hom-module structure.

Definition 9. Let $(H, \alpha)$ be a Hom-Hopf algebra and $(B, \beta)$ a right $\mathrm{H}$-Hom-module coalgebra. Denote by $\mathrm{C}=\mathrm{B} / \mathrm{BH}^{+}$; if there exists a right $H$-Hom-module map $\phi: B \rightarrow H$ which is convolution invertible, then $B / C$ is said to be a cleft coextension and write $(B, \cdot, \phi, \beta)$.

By Proposition 8, we get the main result of this section, which is the generalization of Theorem 2.10 in [8].

Theorem 10. The coextension $B / C$ is cleft if and only if $B$ is isomorphic to a Hom-crossed coproduct $\mathrm{C} \rtimes_{\lambda} \mathrm{H}$ with invertible cocycle $\lambda$.

Proof. If $B$ is isomorphic to the Hom-crossed coproduct $C \rtimes_{\lambda} H$, we prove that the coextension $B / C$ is cleft. First, we show that $\phi: C \rtimes_{\lambda} H \rightarrow H$ is a right $H$-Hom-module map. For any $h, g \in H$, we get

$$
\phi(c \otimes h) \cdot g=\phi(c \otimes h) g=\varepsilon(c) \alpha^{-1}(h) g .
$$

On the other hand,

$$
\begin{aligned}
\phi((c \otimes h) \cdot g) & =\phi\left(c \cdot g_{1} \otimes h g_{2}\right)=\varepsilon\left(c \cdot g_{1}\right) \alpha^{-1}\left(h g_{2}\right) \\
& =\varepsilon(c) \alpha^{-1}(h) g,
\end{aligned}
$$

and by Proposition $8, \lambda$ is an invertible cocycle, so $\phi$ is convolution invertible. Hence $C \rtimes_{\lambda} H / C$ is cleft, so $B / C$ is cleft too.

Conversely, if the coextension $B / C$ is cleft, let us denote by $\bar{c}$ the image in $C$ of the element $c \in B$. Define a weak coaction of $H$ on $C$ by

$$
\begin{aligned}
& \rho: C \longrightarrow H \otimes C, \\
& \rho(\bar{c})=\alpha^{-2}\left(\phi\left(c_{11}\right)\right) \alpha^{-1}\left(\phi^{-1}\left(c_{2}\right)\right) \otimes \beta^{-1}\left(\bar{c}_{12}\right) .
\end{aligned}
$$

The definition is well defined. The conditions (W1), (W2), and (W3) hold which can be directly verified.

Define $\lambda: C \rightarrow H \otimes H$, which is given by

$$
\begin{aligned}
\lambda(\bar{c})= & \alpha^{-3}\left(\phi\left(c_{11}\right)\right) \alpha^{-3}\left(\phi^{-1}\left(c_{2}\right)_{1}\right) \\
& \otimes \alpha^{-3}\left(\phi\left(c_{12}\right)\right) \alpha^{-3}\left(\phi^{-1}\left(c_{2}\right)_{2}\right),
\end{aligned}
$$

and $\omega: C \rightarrow H \otimes H$ is given by

$$
\begin{aligned}
\omega(\bar{c})= & \alpha^{-4}\left(\phi\left(c_{11}\right)_{1}\right) \alpha^{-2}\left(\phi^{-1}\left(c_{2}\right)\right) \\
& \otimes \alpha^{-4}\left(\phi\left(c_{11}\right)_{2}\right) \alpha^{-3}\left(\phi^{-1}\left(c_{12}\right)\right) .
\end{aligned}
$$

Similar to the proof of Proposition 8 , we can obtain that $\lambda *$ $\omega=\omega * \lambda=1$, that is, $\omega$ is the convolution inverse of $\lambda$. With convolution inverse $\lambda$ and weak coaction of $H$ on $C$ as the above, we can define a comultiplication $\Delta$ and a counit $\varepsilon$ as the Definition 2. The comultiplication $\Delta$ is given by

$$
\begin{aligned}
& \Delta(\bar{c} \otimes h)=\bar{c}_{1} \otimes\left(\alpha^{-4}\left(\bar{c}_{21-1}\right) \alpha^{-3}\left(\lambda_{1}\left(\bar{c}_{22}\right)\right)\right) \alpha^{-1}\left(h_{1}\right) \\
& \otimes \beta^{-2}\left(\bar{c}_{210}\right) \otimes \alpha^{-2}\left(\lambda_{2}\left(\bar{c}_{22}\right)\right) \alpha^{-1}\left(h_{2}\right)=\bar{c}_{1} \\
& \otimes\left(\left(\alpha^{-6}\left(\phi\left(c_{2111}\right)\right) \alpha^{-5}\left(\phi^{-1}\left(c_{212}\right)\right)\right)\right.
\end{aligned}
$$




$$
\begin{aligned}
& \left.\cdot\left(\alpha^{-6}\left(\phi\left(c_{2211}\right)\right) \alpha^{-6}\left(\phi^{-1}\left(c_{222}\right)_{1}\right)\right)\right) \alpha^{-1}\left(h_{1}\right) \\
& \otimes \beta^{-3}\left(\bar{c}_{2112}\right) \otimes\left(\alpha^{-5}\left(\phi\left(c_{2212}\right)\right) \alpha^{-5}\left(\phi^{-1}\left(c_{222}\right)_{2}\right)\right) \\
& \cdot \alpha^{-1}\left(h_{2}\right)
\end{aligned}
$$

and the counit $\varepsilon$ is given by

$$
\varepsilon(\bar{c} \otimes h)=\varepsilon(c) \varepsilon(h),
$$

for any $c \in B, h \in H$.

Recalling from [16], the isomorphism is given by

$$
\begin{aligned}
& F: B \longrightarrow C \otimes H, \\
& F(c)=\bar{c}_{1} \otimes \phi\left(c_{2}\right),
\end{aligned}
$$

with inverse

$$
F^{-1}(\bar{c} \otimes h)=c_{(1)} \cdot \phi^{-1}\left(c_{(2)}\right) h .
$$

Now, for any $c \in B, h \in H$, we have

$$
\begin{aligned}
\Delta F & (c)=\Delta\left(\bar{c}_{1} \otimes \phi\left(c_{2}\right)\right)=\bar{c}_{11} \\
& \otimes\left(\left(\alpha^{-6}\left(\phi\left(c_{12111}\right)\right) \alpha^{-5}\left(\phi^{-1}\left(c_{1212}\right)\right)\right)\right. \\
& \left.\cdot\left(\alpha^{-6}\left(\phi\left(c_{12211}\right)\right) \alpha^{-6}\left(\phi^{-1}\left(c_{1222}\right)_{1}\right)\right)\right) \\
& \cdot \alpha^{-1}\left(\phi\left(c_{2}\right)_{1}\right) \otimes \beta^{-3}\left(\bar{c}_{12112}\right) \otimes\left(\alpha^{-5}\left(\phi\left(c_{12212}\right)\right)\right. \\
& \left.\cdot \alpha^{-5}\left(\phi^{-1}\left(c_{1222}\right)_{2}\right)\right) \alpha^{-1}\left(\phi\left(c_{2}\right)_{2}\right)=\bar{c}_{11} \\
& \otimes\left(\alpha^{-4}\left(\phi\left(c_{1211}\right)\right) \varepsilon\left(c_{1221}\right) \alpha^{-6}\left(\phi^{-1}\left(c_{12222}\right)_{1}\right)\right) \\
& \cdot \alpha^{-1}\left(\phi\left(c_{2}\right)_{1}\right) \otimes \beta^{-2}\left(\bar{c}_{1212}\right) \otimes\left(\alpha^{-5}\left(\phi\left(c_{12221}\right)\right)\right. \\
\cdot & \left.\alpha^{-6}\left(\phi^{-1}\left(c_{12222}\right)_{2}\right)\right) \alpha^{-1}\left(\phi\left(c_{2}\right)_{2}\right)=\bar{c}_{11} \\
& \otimes\left(\alpha^{-4}\left(\phi\left(c_{1211}\right)\right) \alpha^{-5}\left(\phi^{-1}\left(c_{1222}\right)_{1}\right)\right) \alpha^{-1}\left(\phi\left(c_{2}\right)_{1}\right) \\
& \otimes \beta^{-2}\left(\bar{c}_{1212}\right) \otimes\left(\alpha^{-4}\left(\phi\left(c_{1221}\right)\right) \alpha^{-5}\left(\phi^{-1}\left(c_{1222}\right)_{2}\right)\right) \\
& \cdot \alpha^{-1}\left(\phi\left(c_{2}\right)_{2}\right)=\alpha^{-1}\left(\bar{c}_{111}\right) \otimes\left(\alpha^{-4}\left(\phi\left(c_{1121}\right)\right)\right. \\
& \left.\cdot \alpha^{-3}\left(\phi^{-1}\left(c_{21}\right)_{1}\right)\right) \alpha^{-2}\left(\phi\left(c_{22}\right)_{1}\right) \otimes \beta^{-2}\left(\bar{c}_{1122}\right) \\
& \otimes\left(\alpha^{-2}\left(\phi\left(c_{12}\right)\right) \alpha^{-3}\left(\phi^{-1}\left(\bar{c}_{21}\right)_{2}\right)\right) \alpha^{-2}\left(\phi\left(c_{22}\right)_{2}\right) \\
& =\alpha^{-1}\left(\bar{c}_{111}\right) \otimes \alpha^{-2}\left(\phi\left(c_{1121}\right)\right) \varepsilon\left(c_{2}\right) \otimes \beta^{-2}\left(\bar{c}_{1122}\right) \\
& \otimes \phi\left(c_{12}\right)=\bar{c}_{11} \otimes \alpha^{-1}\left(\phi\left(c_{121}\right)\right) \otimes \beta^{-1}\left(\bar{c}_{122}\right) \\
& \otimes \alpha\left(\phi\left(c_{2}\right)\right)=\bar{c}_{11} \otimes \phi\left(c_{12}\right) \otimes \bar{c}_{21} \otimes \phi\left(c_{22}\right)=(F \\
& \otimes F) \Delta(c) .
\end{aligned}
$$

then

(1) $(B, \rho, \beta)$ is a right $H$-Hom-comodule;

(2) $(B, \cdot, \rho, \beta)$ is a right H-Hom-Hopf module.

Proof. Note that $\phi$ is convolution invertible right $H$-Hommodule map, where the convolution inverse of $\phi$ is denoted by $\phi^{-1}$. We can get

$$
h_{1} \alpha^{-1} \phi^{-1}\left(b \cdot h_{2}\right)=\varepsilon(h) \alpha\left(\phi^{-1}(b)\right) .
$$

In fact

$$
\begin{aligned}
\varepsilon(h) & \phi^{-1}\left(\beta^{5}(b)\right)=\phi^{-1}\left(\beta^{4}\left(b_{1}\right)\right) \varepsilon\left(b_{2}\right) \varepsilon(h) \\
= & \phi^{-1}\left(\beta^{3}\left(b_{1}\right)\right)\left(\phi * \phi^{-1}\left(b_{2} \cdot h\right)\right) \\
= & \phi^{-1}\left(\beta^{3}\left(b_{1}\right)\right)\left(\phi\left(b_{21} \cdot h_{1}\right) \phi^{-1}\left(b_{22} \cdot h_{2}\right)\right) \\
= & \left(\phi^{-1}\left(\beta^{2}\left(b_{1}\right)\right)\left(\phi\left(b_{21}\right) h_{1}\right)\right) \alpha\left(\phi^{-1}\left(b_{22} \cdot h_{2}\right)\right) \\
= & \left(\left(\phi^{-1}\left(\beta\left(b_{1}\right)\right) \phi\left(b_{21}\right)\right) \alpha\left(h_{1}\right)\right) \alpha\left(\phi^{-1}\left(b_{22} \cdot h_{2}\right)\right) \\
= & \left(\left(\phi^{-1}\left(b_{11}\right)\right) \phi\left(b_{12}\right)\right) \alpha\left(h_{1}\right) \alpha\left(\phi^{-1}\left(\beta\left(b_{2}\right) \cdot h_{2}\right)\right)
\end{aligned}
$$

So we can obtain that $(F \otimes F) \Delta=\Delta F$ and $\varepsilon=\varepsilon F$. Since $B$ is a Hom-coalgebra, $C \otimes H$ is a Hom-crossed coproduct and $F$ is the required Hom-coalgebra isomorphism. Thus the proof is complete.

Now we give a lemma, which will be used in the sequel.
Lemma 11. Let $(H, \alpha)$ be a Hom-Hopf algebra and $(B, \cdot, \rho, \beta)$ for any $a \in A$. With the above defined comultiplication, we can prove that $A$ is a Hom-coalgebra. Furthermore we can get that $\psi$, which is as similar as defined in (1), is also a Hom-

Then we discuss the relation between cleft coextension and Hom-module coalgebra with the Hom-Hopf module

Proposition 12. Let $(B, \cdot, \phi, \beta)$ be a cleft coextension. One

$$
\begin{aligned}
\rho(b)= & \beta^{-2}\left(b_{11}\right) \cdot \alpha^{-3}\left(\phi^{-1}\left(b_{12}\right) \phi\left(b_{2}\right)_{1}\right) \\
& \otimes \alpha^{-1}\left(\phi\left(b_{2}\right)_{2}\right),
\end{aligned}
$$




$$
\begin{aligned}
& =\varepsilon\left(b_{1}\right) \alpha^{2}\left(h_{1}\right) \alpha\left(\phi^{-1}\left(\beta\left(b_{2}\right) \cdot h_{2}\right)\right) \\
& =\alpha^{2}\left(h_{1}\right) \alpha\left(\phi^{-1}\left(\beta^{2}(b) \cdot h_{2}\right)\right) .
\end{aligned}
$$

(1) Now we prove that $(B, \rho, \beta)$ is a right $H$-Homcomodule. For any $b \in B$,

$$
\begin{aligned}
& (\rho \otimes \alpha) \rho(b)=(\rho \otimes \alpha)\left(\beta^{-2}\left(b_{11}\right)\right. \\
& \left.\cdot \alpha^{-3}\left(\phi^{-1}\left(b_{12}\right) \phi\left(b_{2}\right)_{1}\right) \otimes \alpha^{-1}\left(\phi\left(b_{2}\right)_{2}\right)\right) \\
& =\left(\beta^{-4}\left(b_{1111}\right) \cdot \alpha^{-5}\left(\phi^{-1}\left(b_{12}\right) \phi\left(b_{2}\right)_{1}\right)_{11}\right) \\
& \cdot \alpha^{-3} \phi^{-1}\left(\beta^{-2}\left(b_{1112}\right) \cdot \alpha^{-3}\left(\phi^{-1}\left(b_{12}\right) \phi\left(b_{2}\right)_{1}\right)_{12}\right) \\
& \cdot \alpha^{-3} \phi\left(\beta^{-2}\left(b_{112}\right)\right. \\
& \left.\cdot \alpha^{-3}\left(\phi^{-1}\left(b_{12}\right) \phi\left(b_{2}\right)_{1}\right)_{2}\right)_{1} \otimes \alpha^{-1} \phi\left(\beta^{-2}\left(b_{112}\right)\right. \\
& \left.\cdot \alpha^{-3}\left(\phi^{-1}\left(b_{12}\right) \phi\left(b_{2}\right)_{1}\right)_{2}\right)_{2} \otimes \phi\left(b_{2}\right)_{2}=\beta^{-3}\left(b_{1111}\right) \\
& \cdot\left(\alpha^{-6}\left(\phi^{-1}\left(b_{12}\right) \phi\left(b_{2}\right)_{1}\right)_{11}\right. \\
& \left.\cdot \alpha^{-4} \phi^{-1}\left(\beta^{-2}\left(b_{1112}\right) \cdot \alpha^{-3}\left(\phi^{-1}\left(b_{12}\right) \phi\left(b_{2}\right)_{1}\right)_{12}\right)\right) \\
& \cdot \alpha^{-3} \phi\left(\beta^{-2}\left(b_{112}\right)\right. \\
& \left.\cdot \alpha^{-3}\left(\phi^{-1}\left(b_{12}\right) \phi\left(b_{2}\right)_{1}\right)_{2}\right)_{1} \otimes \alpha^{-1} \phi\left(\beta^{-2}\left(b_{112}\right)\right. \\
& \left.\cdot \alpha^{-3}\left(\phi^{-1}\left(b_{12}\right) \phi\left(b_{2}\right)_{1}\right)_{2}\right)_{2} \otimes \phi\left(b_{2}\right)_{2} \\
& \stackrel{(65)}{=} \beta^{-3}\left(b_{1111}\right) \cdot \varepsilon\left(\left(\phi^{-1}\left(b_{12}\right) \phi\left(b_{2}\right)_{1}\right)_{1}\right) \\
& \cdot \phi^{-1}\left(\beta^{-4}\left(b_{1112}\right)\right) \alpha^{-3} \phi\left(\beta^{-2}\left(b_{112}\right)\right. \\
& \left.\cdot \alpha^{-3}\left(\phi^{-1}\left(b_{12}\right) \phi\left(b_{2}\right)_{1}\right)_{2}\right)_{1} \otimes \alpha^{-1} \phi\left(\beta^{-2}\left(b_{112}\right)\right. \\
& \left.\cdot \alpha^{-3}\left(\phi^{-1}\left(b_{12}\right) \phi\left(b_{2}\right)_{1}\right)_{2}\right)_{2} \otimes \phi\left(b_{2}\right)_{2}=\beta^{-3}\left(b_{1111}\right) \\
& \cdot \phi^{-1}\left(\beta^{-4}\left(b_{1112}\right)\right) \alpha^{-3} \phi\left(\beta^{-2}\left(b_{112}\right)\right. \\
& \left.\cdot \alpha^{-2}\left(\phi^{-1}\left(b_{12}\right) \phi\left(b_{2}\right)_{1}\right)\right)_{1} \otimes \alpha^{-1} \phi\left(\beta^{-2}\left(b_{112}\right)\right. \\
& \left.\cdot \alpha^{-2}\left(\phi^{-1}\left(b_{12}\right) \phi\left(b_{2}\right)_{1}\right)\right)_{2} \otimes \phi\left(b_{2}\right)_{2}=\beta^{-3}\left(b_{1111}\right) \\
& \cdot \phi^{-1}\left(\beta^{-4}\left(b_{1112}\right)\right)\left(\alpha^{-5} \phi\left(b_{112}\right)\right. \\
& \left.\cdot \alpha^{-5}\left(\phi^{-1}\left(b_{12}\right) \phi\left(b_{2}\right)_{1}\right)\right)_{1} \otimes\left(\alpha^{-3} \phi\left(b_{112}\right)\right. \\
& \left.\cdot \alpha^{-3}\left(\phi^{-1}\left(b_{12}\right) \phi\left(b_{2}\right)_{1}\right)\right)_{2} \otimes \phi\left(b_{2}\right)_{2}=\beta^{-3}\left(b_{1111}\right) \\
& \cdot \phi^{-1}\left(\beta^{-4}\left(b_{1112}\right)\right)\left(\left(\alpha^{-6}\left(\phi\left(b_{112}\right)\right) \alpha^{-5}\left(\phi^{-1}\left(b_{12}\right)\right)\right)\right. \\
& \left.\cdot \alpha^{-4} \phi\left(b_{2}\right)_{1}\right)_{1} \otimes\left(\left(\alpha^{-4}\left(\phi\left(b_{112}\right)\right) \alpha^{-3}\left(\phi^{-1}\left(b_{12}\right)\right)\right)\right. \\
& \left.\cdot \alpha^{-2} \phi\left(b_{2}\right)_{1}\right)_{2} \otimes \phi\left(b_{2}\right)_{2}=\beta^{-1}\left(b_{11}\right) \\
& \cdot \phi^{-1}\left(\beta^{-2}\left(b_{12}\right)\right) \varepsilon\left(b_{21}\right) \alpha^{-4}\left(\phi\left(b_{22}\right)_{11}\right)
\end{aligned}
$$

$$
\begin{aligned}
& \otimes \alpha^{-2}\left(\phi\left(b_{22}\right)_{12}\right) \otimes \phi\left(b_{2}\right)_{2}=\beta^{-1}\left(b_{11}\right) \\
& \cdot \phi^{-1}\left(\beta^{-2}\left(b_{12}\right)\right) \alpha^{-3}\left(\phi\left(b_{2}\right)_{11}\right) \otimes \alpha^{-1}\left(\phi\left(b_{2}\right)_{12}\right) \\
& \otimes \phi\left(b_{2}\right)_{2}=\beta^{-1}\left(b_{11}\right) \cdot \phi^{-1}\left(\beta^{-2}\left(b_{12}\right)\right) \alpha^{-2}\left(\phi\left(b_{2}\right)_{1}\right) \\
& \otimes \alpha^{-1}\left(\phi\left(b_{2}\right)_{21}\right) \otimes \alpha^{-1}\left(\phi\left(b_{2}\right)_{22}\right)=(\alpha \otimes \Delta) \rho(b),
\end{aligned}
$$

and $(\beta \otimes \varepsilon) \rho(b)=b$; thus $(B, \rho, \beta)$ is a right $H$-Homcomodule.

(2) For all $b \in B$ and $h \in H$, we can calculate

$$
\begin{aligned}
& \rho(b \cdot h)=\beta^{-2}\left((b \cdot h)_{11}\right) \\
& \cdot \alpha^{-3}\left(\phi^{-1}\left((b \cdot h)_{12}\right) \phi\left((b \cdot h)_{2}\right)_{1}\right) \\
& \otimes \alpha^{-1}\left(\phi\left((b \cdot h)_{2}\right)_{2}\right)=\beta^{-2}\left(b_{11} \cdot h_{11}\right) \\
& \cdot \alpha^{-3}\left(\phi^{-1}\left(b_{12} \cdot h_{12}\right) \phi\left(b_{2} \cdot h_{2}\right)_{1}\right) \\
& \otimes \alpha^{-1}\left(\phi\left(b_{2} \cdot h_{2}\right)_{2}\right)=\beta^{-1}\left(b_{11}\right) \\
& \cdot\left(\alpha^{-3}\left(h_{11}\right) \alpha^{-4}\left(\phi^{-1}\left(b_{12} \cdot h_{12}\right)\right)\right) \alpha^{-3}\left(\phi\left(b_{2} \cdot h_{2}\right)_{1}\right) \\
& \otimes \alpha^{-1}\left(\phi\left(b_{2} \cdot h_{2}\right)_{2}\right) \stackrel{(65)}{=} \beta^{-1}\left(b_{11}\right) \\
& \cdot \varepsilon\left(h_{1}\right) \alpha^{-2}\left(\phi^{-1}\left(b_{12}\right)\right) \alpha^{-3}\left(\phi\left(b_{2} \cdot h_{2}\right)_{1}\right) \\
& \otimes \alpha^{-1}\left(\phi\left(b_{2} \cdot h_{2}\right)_{2}\right)=\beta^{-1}\left(b_{11}\right) \\
& \cdot \alpha^{-2}\left(\phi^{-1}\left(b_{12}\right)\left(\alpha^{-3}\left(\phi\left(b_{2}\right)_{1}\right)\right) \alpha^{-2}(h)_{1}\right) \\
& \otimes \alpha^{-1}\left(\phi\left(b_{2}\right)_{2}\right) h_{2} \\
& =\left(\beta^{-2}\left(b_{11}\right) \cdot \alpha^{-3}\left(\phi^{-1}\left(b_{12}\right) \phi\left(b_{2}\right)_{1}\right)\right) \cdot h_{1} \\
& \otimes \alpha^{-1}\left(\phi\left(b_{2}\right)_{2}\right) h_{2}=b_{(0)} h_{1} \otimes b_{(1)} h_{1} ;
\end{aligned}
$$

thus $(B, \cdot, \rho, \beta)$ is a right $H$-Hom-Hopf module.

Theorem 13. Let $(H, \alpha)$ be a Hom-Hopf algebra and $(B, \cdot, \beta)$ a right $\mathrm{H}$-Hom-module coalgebra, if $\mathrm{C}=\mathrm{B} / \mathrm{BH}^{+}$, then $\mathrm{B} / \mathrm{C}$ is a cleft coextension if and only if $(B, \cdot, \rho, \beta)$ is a right $\mathrm{H}$-HomHopf module, and there exists a convolution invertible linear map $\phi: B \rightarrow H$ satisfying $\phi(b)=\varepsilon\left(b_{(0)}\right) \beta^{-1}\left(b_{(1)}\right)$.

Proof. Assume that $B$ is a cleft coextension, then there exists a convolution invertible Hom-module map $\phi: B \rightarrow H$ such that $\varepsilon \phi=\varepsilon$, so we have $\varepsilon \phi^{-1}=\varepsilon$. Define a map,

$$
\begin{aligned}
\rho: B & \longrightarrow B \otimes H \\
\rho(b)= & \beta^{-2}\left(b_{11}\right) \cdot \alpha^{-3}\left(\phi^{-1}\left(b_{12}\right) \phi\left(b_{2}\right)_{1}\right) \\
& \otimes \alpha^{-1}\left(\phi\left(b_{2}\right)_{2}\right),
\end{aligned}
$$

$\forall b \in B$

clearly, $\phi(b)=\varepsilon\left(b_{(0)}\right) \beta^{-1}\left(b_{(1)}\right)$. By Proposition 12 , we see that $(B, \cdot \rho, \beta)$ is a right $H$-Hom-Hopf module. 
Conversely, it is sufficient to show that $\phi$ is a right $H$ Hom-module map. Using the fact that $(B, \cdot, \rho, \beta)$ is a right $H$ Hom-Hopf module and $\phi(b)=\varepsilon\left(b_{(0)}\right) \beta^{-1}\left(b_{(1)}\right)$, for any $b \in B$ and $h \in H$, we get

$$
\begin{aligned}
\phi(b \cdot h) & =\varepsilon\left((b \cdot h)_{(0)}\right) \beta^{-1}\left((b \cdot h)_{(1)}\right) \\
& =\varepsilon\left(b_{(0)}\right) \varepsilon\left(h_{1}\right) \beta^{-1}\left(b_{(1)}\right) \alpha^{-1}\left(h_{2}\right)=\phi(b) h .
\end{aligned}
$$

The proof is complete.

\section{The Factorization of Hom-Module Coalgebra with Hopf Module Structure}

In this section, we always assume that $(H, \alpha)$ is a Hom-Hopf algebra and $(B, \cdot, \phi, \beta)$ is a cleft coextension. Based on this assumption, $B$ can not only form a right $H$-Hom-comodule, but also form a right $H$-Hom-Hopf module. Furthermore, we obtain the factorization of Hom-coalgebra $B$.

Now, we can obtain a Hom-coalgebra factorization for Hom-module coalgebra with the Hom-Hopf module structure.

Theorem 14. Let $(H, \alpha)$ be a Hom-Hopf algebra and $(B, \cdot, \rho, \beta)$ a right $\mathrm{H}$-Hom-Hopf module. One assumes that $(B, \cdot, \beta)$ is a right $H$-Hom-module coalgebra and set $A=B^{c o H}=\{a \in B \mid$ $\rho(a)=\beta(a) \otimes 1\}$. The comultiplication on $A \otimes H$ can be defined

$$
\begin{aligned}
\Delta(a \otimes h)= & \beta^{-3}\left(a_{1(0)(0)}\right) \cdot S\left(\alpha^{-3}\left(a_{1(0)(1)}\right)\right) \\
& \otimes \alpha^{-2}\left(a_{1(1)}\right) \alpha^{-1}\left(h_{1}\right) \otimes \beta^{-3}\left(a_{2(0)(0)}\right) \\
& \cdot S\left(\alpha^{-3}\left(a_{2(0)(1)}\right)\right) \otimes \alpha^{-2}\left(a_{2(1)}\right) \alpha^{-1}\left(h_{2}\right),
\end{aligned}
$$

and the counit can be defined

$$
\varepsilon(a \otimes h)=\varepsilon(a) \varepsilon(h),
$$

for any $a \in A, h \in H$, then $(A \otimes H, \alpha \otimes \beta)$ is a Hom-coalgebra, and one writes it as $A \square \mathrm{\square}$. Moreover, $B \simeq A \square H$ as Homcoalgebras.

Proof. Since $(B, \cdot, \rho)$ is a right $H$-Hom-Hopf module, $\Delta$ is well defined and the map

$$
\begin{aligned}
\varphi: A \otimes H & \longrightarrow B, \\
a \otimes h & \longmapsto a \cdot h
\end{aligned}
$$

is an isomorphism of right $H$-Hom-Hopf module.

We first verify that $(A \otimes H, \Delta, \varepsilon, \beta \otimes \alpha)$ is a Hom-coalgebra. It is easy to see that $(\varepsilon \otimes(\beta \otimes \alpha)) \Delta=((\beta \otimes \alpha) \otimes \varepsilon) \Delta$. It is sufficient to show the coassociativity. For any $a \in A, h \in H$, we calculate as follows:

$$
\begin{aligned}
(\Delta & \otimes(\beta \otimes \alpha)) \Delta(a \otimes h) \\
= & \left(\beta^{-6}\left(a_{1(0)(0) 1(0)(0)}\right) \cdot S\left(\alpha^{-6}\left(a_{1(0)(1) 222}\right)\right)\right) \\
\cdot & S\left(\alpha^{-6}\left(a_{1(0)(0) 1(0)(1)}\right) S\left(\alpha^{-6}\left(a_{1(0)(1) 221}\right)\right)\right)
\end{aligned}
$$

$$
\begin{aligned}
& \otimes\left(\alpha^{-5}\left(a_{1(0)(0) 1(1)}\right) S\left(\alpha^{-5}\left(a_{1(0)(1) 21}\right)\right)\right) \\
& \cdot\left(\alpha^{-3}\left(a_{1(1) 1}\right) \alpha^{-2}\left(h_{11}\right)\right) \\
& \otimes\left(\beta^{-6}\left(a_{1(0)(0) 2(0)(0)}\right) \cdot S\left(\alpha^{-6}\left(a_{1(0)(1) 122}\right)\right)\right) \\
& \cdot S\left(\alpha^{-6}\left(a_{1(0)(0) 2(0)(1)}\right) S\left(\alpha^{-6}\left(a_{1(0)(1) 121}\right)\right)\right) \\
& \otimes\left(\alpha^{-5}\left(a_{1(0)(0) 2(1)}\right) S\left(\alpha^{-5}\left(a_{1(0)(1) 11}\right)\right)\right) \\
& \cdot\left(\alpha^{-3}\left(a_{1(1) 2}\right) \alpha^{-2}\left(h_{12}\right)\right) \otimes \beta^{-2}\left(a_{2(0)(0)}\right) \\
& \cdot S\left(\alpha^{-2}\left(a_{2(0)(1)}\right)\right) \otimes \alpha^{-1}\left(a_{2(1)}\right) h_{2} \\
& =\beta^{-5}\left(a_{1(0)(0) 1(0)(0)}\right) \\
& \cdot S\left(\alpha^{-6}\left(a_{1(0)(0) 1(0)(1)}\right) \varepsilon\left(a_{1(0)(1) 22}\right) 1_{H}\right) \\
& \otimes\left(\alpha^{-5}\left(a_{1(0)(0) 1(1)}\right) S\left(\alpha^{-5}\left(a_{1(0)(1) 21}\right)\right)\right) \\
& \cdot\left(\alpha^{-3}\left(a_{1(1) 1}\right) \alpha^{-2}\left(h_{11}\right)\right) \otimes \beta^{-5}\left(a_{1(0)(0) 2(0)(0)}\right) \\
& \cdot S\left(\alpha^{-6}\left(a_{1(0)(0) 2(0)(1)}\right) \varepsilon\left(a_{1(0)(1) 12}\right) 1_{H}\right) \\
& \otimes\left(\alpha^{-5}\left(a_{1(0)(0) 2(1)}\right) S\left(\alpha^{-5}\left(a_{1(0)(1) 11}\right)\right)\right) \\
& \cdot\left(\alpha^{-3}\left(a_{1(1) 2}\right) \alpha^{-2}\left(h_{12}\right)\right) \otimes \beta^{-2}\left(a_{2(0)(0)}\right) \\
& \cdot S\left(\alpha^{-2}\left(a_{2(0)(1)}\right)\right) \otimes \alpha^{-1}\left(a_{2(1)}\right) h_{2} \\
& =\beta^{-5}\left(a_{1(0)(0) 1(0)(0)}\right) \cdot S\left(\alpha^{-5}\left(a_{1(0)(0) 1(0)(1)}\right)\right) \\
& \otimes\left(\alpha^{-5}\left(a_{1(0)(0) 1(1)}\right) S\left(\alpha^{-4}\left(a_{1(0)(1) 2}\right)\right)\right) \\
& \cdot\left(\alpha^{-3}\left(a_{1(1) 1}\right) \alpha^{-2}\left(h_{11}\right)\right) \otimes \beta^{-5}\left(a_{1(0)(0) 2(0)(0)}\right) \\
& \cdot S\left(\alpha^{-5}\left(a_{1(0)(0) 2(0)(1)}\right)\right) \\
& \otimes\left(\alpha^{-5}\left(a_{1(0)(0) 2(1)}\right) S\left(\alpha^{-4}\left(a_{1(0)(1) 1}\right)\right)\right) \\
& \cdot\left(\alpha^{-3}\left(a_{1(1) 2}\right) \alpha^{-2}\left(h_{12}\right)\right) \otimes \beta^{-2}\left(a_{2(0)(0)}\right) \\
& \cdot S\left(\alpha^{-2}\left(a_{2(0)(1)}\right)\right) \otimes \alpha^{-1}\left(a_{2(1)}\right) h_{2} \\
& =\beta^{-4}\left(a_{1(0) 1(0)(0)}\right) \cdot S\left(\alpha^{-4}\left(a_{1(0) 1(0)(1)}\right)\right) \\
& \otimes\left(\alpha^{-4}\left(a_{1(0) 1(1)}\right) S\left(\alpha^{-4}\left(a_{1(1) 12}\right)\right)\right) \\
& \cdot\left(\alpha^{-4}\left(a_{1(1) 21}\right) \alpha^{-2}\left(h_{11}\right)\right) \otimes \beta^{-4}\left(a_{1(0) 2(0)(0)}\right) \\
& \text { · } S\left(\alpha^{-4}\left(a_{1(0) 2(0)(1)}\right)\right) \\
& \otimes\left(\alpha^{-4}\left(a_{1(0) 2(1)}\right) S\left(\alpha^{-4}\left(a_{1(1) 11}\right)\right)\right) \\
& \cdot\left(\alpha^{-4}\left(a_{1(1) 22}\right) \alpha^{-2}\left(h_{12}\right)\right) \otimes \beta^{-2}\left(a_{2(0)(0)}\right) \\
& \cdot S\left(\alpha^{-2}\left(a_{2(0)(1)}\right)\right) \otimes \alpha^{-1}\left(a_{2(1)}\right) h_{2}
\end{aligned}
$$


$=\beta^{-4}\left(a_{1(0) 1(0)(0)}\right) \cdot S\left(\alpha^{-4}\left(a_{1(0) 1(0)(1)}\right)\right)$

$\otimes \alpha^{-3}\left(a_{1(0) 1(1)}\right)$

$\cdot\left(\left(S\left(\alpha^{-5}\left(a_{1(1) 12}\right)\right) \alpha^{-5}\left(a_{1(1) 21}\right)\right) \alpha^{-2}\left(h_{11}\right)\right)$

$\otimes \beta^{-4}\left(a_{1(0) 2(0)(0)}\right) \cdot S\left(\alpha^{-4}\left(a_{1(0) 2(0)(1)}\right)\right)$

$\otimes \alpha^{-3}\left(a_{1(0) 2(1)}\right)$

$\cdot\left(\left(S\left(\alpha^{-5}\left(a_{1(1) 11}\right)\right) \alpha^{-5}\left(a_{1(1) 22}\right)\right) \alpha^{-2}\left(h_{12}\right)\right)$

$\otimes \beta^{-2}\left(a_{2(0)(0)}\right) \cdot S\left(\alpha^{-2}\left(a_{2(0)(1)}\right)\right) \otimes \alpha^{-1}\left(a_{2(1)}\right) h_{2}$

$=\beta^{-4}\left(a_{1(0) 1(0)(0)}\right) \cdot S\left(\alpha^{-4}\left(a_{1(0) 1(0)(1)}\right)\right)$

$\otimes \alpha^{-3}\left(a_{1(0) 1(1)}\right) \varepsilon\left(a_{1(1) 21}\right) \alpha^{-1}\left(h_{11}\right)$

$\otimes \beta^{-4}\left(a_{1(0) 2(0)(0)}\right) \cdot S\left(\alpha^{-4}\left(a_{1(0) 2(0)(1)}\right)\right)$

$\otimes \alpha^{-3}\left(a_{1(0) 2(1)}\right)$

$\cdot\left(\left(S\left(\alpha^{-4}\left(a_{1(1) 1}\right)\right) \alpha^{-5}\left(a_{1(1) 22}\right)\right) \alpha^{-2}\left(h_{12}\right)\right)$

$\otimes \beta^{-2}\left(a_{2(0)(0)}\right) \cdot S\left(\alpha^{-2}\left(a_{2(0)(1)}\right)\right) \otimes \alpha^{-1}\left(a_{2(1)}\right) h_{2}$

$=\beta^{-4}\left(a_{1(0) 1(0)(0)}\right) \cdot S\left(\alpha^{-4}\left(a_{1(0) 1(0)(1)}\right)\right)$

$\otimes \alpha^{-3}\left(a_{1(0) 1(1)}\right) \alpha^{-1}\left(h_{11}\right) \otimes \beta^{-4}\left(a_{1(0) 2(0)(0)}\right)$

$\cdot S\left(\alpha^{-4}\left(a_{1(0) 2(0)(1)}\right)\right) \otimes \alpha^{-3}\left(a_{1(0) 2(1)}\right)$

$\cdot\left(\left(S\left(\alpha^{-4}\left(a_{1(1) 1}\right)\right) \alpha^{-4}\left(a_{1(1) 2}\right)\right) \alpha^{-2}\left(h_{12}\right)\right)$

$\otimes \beta^{-2}\left(a_{2(0)(0)}\right) \cdot S\left(\alpha^{-2}\left(a_{2(0)(1)}\right)\right) \otimes \alpha^{-1}\left(a_{2(1)}\right) h_{2}$

$=\beta^{-4}\left(a_{1(0) 1(0)(0)}\right) \cdot S\left(\alpha^{-4}\left(a_{1(0) 1(0)(1)}\right)\right)$

$\otimes \alpha^{-3}\left(a_{1(0) 1(1)}\right) \alpha^{-1}\left(h_{11}\right) \otimes \beta^{-4}\left(a_{1(0) 2(0)(0)}\right)$

$\cdot S\left(\alpha^{-4}\left(a_{1(0) 2(0)(1)}\right)\right) \otimes \alpha^{-3}\left(a_{1(0) 2(1)}\right) \varepsilon\left(a_{1(1)}\right)$

$\cdot \alpha^{-1}\left(h_{12}\right) \otimes \beta^{-2}\left(a_{2(0)(0)}\right) \cdot S\left(\alpha^{-2}\left(a_{2(0)(1)}\right)\right)$

$\otimes \alpha^{-1}\left(a_{2(1)}\right) h_{2}=\beta^{-3}\left(a_{11(0)(0)}\right) \cdot S\left(\alpha^{-3}\left(a_{11(0)(1)}\right)\right)$

$\otimes \alpha^{-2}\left(a_{11(1)}\right) \alpha^{-1}\left(h_{11}\right) \otimes \beta^{-3}\left(a_{12(0)(0)}\right)$

$\cdot S\left(\alpha^{-3}\left(a_{12(0)(1)}\right)\right) \otimes \alpha^{-2}\left(a_{12(1)}\right) \alpha^{-1}\left(h_{12}\right)$

$\otimes \beta^{-2}\left(a_{2(0)(0)}\right) \cdot S\left(\alpha^{-2}\left(a_{2(0)(1)}\right)\right) \otimes \alpha^{-1}\left(a_{2(1)}\right) h_{2}$.

In a similar way

$((\beta \otimes \alpha) \otimes \Delta) \Delta(a \otimes h)$

$$
\begin{aligned}
= & \beta^{-2}\left(a_{1(0)(0)}\right) \cdot S\left(\alpha^{-2}\left(a_{1(0)(0)}\right)\right) \otimes \alpha^{-1}\left(a_{1(1)}\right) h_{1} \\
& \otimes \beta^{-3}\left(a_{21(0)(0)}\right) \cdot S\left(\alpha^{-3}\left(a_{21(0)(1)}\right)\right)
\end{aligned}
$$

$$
\begin{aligned}
& \otimes \alpha^{-2}\left(a_{21(1)}\right) \alpha^{-1}\left(h_{21}\right) \otimes \beta^{-3}\left(a_{22(0)(0)}\right) \\
& \cdot S\left(\alpha^{-3}\left(a_{22(0)(1)}\right)\right) \otimes \alpha^{-2}\left(a_{22(1)}\right) \alpha^{-1}\left(h_{22}\right) .
\end{aligned}
$$

Thus, $(A \otimes H, \Delta, \varepsilon, \beta \otimes \alpha)$ is a Hom-coalgebra.

Next we show that $\varphi$ is a Hom-coalgebra morphism.

$$
\begin{aligned}
\varphi\left((a \otimes h)_{1}\right) \otimes \varphi\left((a \otimes h)_{2}\right) \\
=\left(\beta^{-3}\left(a_{1(0)(0)}\right) \cdot S\left(\alpha^{-3}\left(a_{1(0)(1)}\right)\right)\right) \\
\cdot \alpha^{-2}\left(a_{1(1)}\right) \alpha^{-1}\left(h_{1}\right) \\
\otimes\left(\beta^{-3}\left(a_{2(0)(0)}\right) \cdot S\left(\alpha^{-3}\left(a_{2(0)(1)}\right)\right)\right) \\
\cdot \alpha^{-2}\left(a_{2(1)}\right) \alpha^{-1}\left(h_{2}\right) \\
=\beta^{-1}\left(a_{1(0)}\right) \\
\cdot\left(\left(S\left(\alpha^{-4}\left(a_{1(1) 1}\right)\right) \alpha^{-4}\left(a_{1(1) 2}\right)\right) \alpha^{-1}\left(h_{1}\right)\right) \\
\otimes \beta^{-1}\left(a_{2(0)}\right) \\
\quad \cdot\left(\left(S\left(\alpha^{-4}\left(a_{2(1) 1}\right)\right) \alpha^{-4}\left(a_{2(1) 2}\right)\right) \alpha^{-1}\left(h_{2}\right)\right) \\
=a_{1} \cdot h_{1} \otimes a_{2} \cdot h_{2}=\varphi\left(a_{1} \otimes h_{1}\right) \otimes \varphi\left(a_{2} \otimes h_{2}\right) .
\end{aligned}
$$

Therefore, $B \simeq A \square H$ as Hom-coalgebras.

By the above results, we obtain the following result.

Theorem 15. Let $(H, \alpha)$ be a Hom-Hopf algebra and $(B, \cdot, \phi, \beta)$ a cleft coextension, then

$$
B \simeq C \rtimes_{\lambda} H \simeq A \square^{\prime} H
$$

as Hom-coalgebras, where $\mathrm{C}=\mathrm{B} / \mathrm{BH}^{+}$, and

$$
\begin{aligned}
A & =\left\{b \in B \mid \beta^{-2}\left(b_{11}\right) \cdot \alpha^{-3}\left(\phi^{-1}\left(b_{12}\right) \phi\left(b_{2}\right)_{1}\right)\right. \\
& \left.\otimes \alpha^{-1}\left(\phi\left(b_{2}\right)_{2}\right)=\beta(b) \otimes 1\right\} .
\end{aligned}
$$

Proof. By Theorems 10 and 14, we have $B \simeq C \rtimes_{\lambda} H$ and $B \simeq$ $A \square H$ as Hom-coalgebras, respectively. Since $(B, \cdot, \phi, \beta)$ is a cleft coextension, we get $(B, \cdot, \rho, \beta)$ is a right $H$-Hom-Hopf module by Proposition 12, where the comodule structure is defined by

$$
\begin{aligned}
\rho(b)= & \beta^{-2}\left(b_{11}\right) \cdot \alpha^{-3}\left(\phi^{-1}\left(b_{12}\right) \phi\left(b_{2}\right)_{1}\right) \\
& \otimes \alpha^{-1}\left(\phi\left(b_{2}\right)_{2}\right) .
\end{aligned}
$$

We know that

$$
\begin{aligned}
\psi: C & \longrightarrow B^{c o H}, \\
\bar{b} & \longmapsto b_{(0)} \cdot S\left(b_{(1)}\right)
\end{aligned}
$$

is an isomorphism of Hom-coalgebras by Lemma 11. Set

$$
\begin{gathered}
\bar{\psi}=\psi \otimes i d, \\
\bar{\psi}: C \rtimes_{\lambda} H \longrightarrow A \square \dot{H} .
\end{gathered}
$$


We only need to verify that $\bar{\psi}$ is a Hom-coalgebra morphism. First we prove that

$$
\psi(\bar{b})=b_{1} \cdot \phi^{-1}\left(b_{2}\right) .
$$

In fact

$$
\begin{aligned}
\psi(\bar{b})= & b_{(0)} \cdot S\left(b_{(1)}\right) \\
= & \beta^{-2}\left(b_{11}\right) \cdot \alpha^{-3}\left(\phi^{-1}\left(b_{12}\right) \phi\left(b_{2}\right)_{1}\right) \\
& \cdot S\left(\alpha^{-1}\left(\phi\left(b_{2}\right)_{2}\right)\right) \\
= & \beta^{-1}\left(b_{11}\right) \cdot \alpha^{-1}\left(\phi^{-1}\left(b_{12}\right)\right) \varepsilon\left(\phi\left(b_{2}\right)\right) \\
= & b_{1} \cdot \phi^{-1}\left(b_{2}\right) .
\end{aligned}
$$

On one hand, we can calculate

$$
\begin{aligned}
& \Delta \bar{\psi}(\bar{b} \otimes h) \stackrel{(83)}{=} \Delta\left(b_{1} \cdot \phi^{-1}\left(b_{2}\right) \otimes h\right) \\
& =\beta^{-3}\left(\left(b_{1} \cdot \phi^{-1}\left(b_{2}\right)\right)_{1(0)(0)}\right) \\
& \cdot S\left(\alpha^{-3}\left(\left(b_{1} \cdot \phi^{-1}\left(b_{2}\right)\right)_{1(0)(1)}\right)\right) \\
& \otimes \alpha^{-2}\left(\left(b_{1} \cdot \phi^{-1}\left(b_{2}\right)\right)_{1(1)}\right) \alpha^{-1}\left(h_{1}\right) \\
& \otimes \beta^{-3}\left(\left(b_{1} \cdot \phi^{-1}\left(b_{2}\right)\right)_{2(0)(0)}\right) \\
& \cdot S\left(\alpha^{-3}\left(\left(b_{1} \cdot \phi^{-1}\left(b_{2}\right)\right)_{2(0)(1)}\right)\right) \\
& \otimes \alpha^{-2}\left(\left(b_{1} \cdot \phi^{-1}\left(b_{2}\right)\right)_{2(1)}\right) \alpha^{-1}\left(h_{2}\right) \\
& =\beta^{-3}\left(b_{11(0)(0)} \cdot \phi^{-1}\left(b_{2}\right)_{111}\right) \\
& \cdot S\left(\alpha^{-3}\left(b_{11(0)(1)} \phi^{-1}\left(b_{2}\right)_{112}\right)\right) \\
& \otimes \alpha^{-2}\left(b_{11(1)} \phi^{-1}\left(b_{2}\right)_{12}\right) \alpha^{-1}\left(h_{1}\right) \\
& \otimes \beta^{-3}\left(b_{12(0)(0)} \cdot \phi^{-1}\left(b_{2}\right)_{211}\right) \\
& \cdot S\left(\alpha^{-3}\left(b_{12(0)(1)} \phi^{-1}\left(b_{2}\right)_{212}\right)\right) \\
& \otimes \alpha^{-2}\left(b_{12(1)} \phi^{-1}\left(b_{2}\right)_{22}\right) \alpha^{-1}\left(h_{2}\right)=\beta^{-2}\left(b_{11(0)(0)}\right) \\
& \cdot\left(\alpha^{-4}\left(\phi^{-1}\left(b_{2}\right)_{111}\right) S\left(\alpha^{-4}\left(\phi^{-1}\left(b_{2}\right)_{112}\right)\right)\right) \\
& \cdot S\left(\alpha^{-3}\left(b_{11(0)(1)}\right)\right) \otimes \alpha^{-2}\left(b_{11(1)} \phi^{-1}\left(b_{2}\right)_{12}\right) \alpha^{-1}\left(h_{1}\right) \\
& \otimes \beta^{-2}\left(b_{12(0)(0)}\right) \\
& \cdot\left(\alpha^{-4}\left(\phi^{-1}\left(b_{2}\right)_{211}\right) S\left(\alpha^{-4}\left(\phi^{-1}\left(b_{2}\right)_{212}\right)\right)\right) \\
& \cdot S\left(\alpha^{-3}\left(b_{12(0)(1)}\right)\right) \otimes \alpha^{-2}\left(b_{12(1)} \phi^{-1}\left(b_{2}\right)_{22}\right) \alpha^{-1}\left(h_{2}\right) \\
& =\beta^{-2}\left(b_{11(0)(0)}\right) \cdot S\left(\alpha^{-2}\left(b_{11(0)(1)}\right)\right) \\
& \otimes\left(\alpha^{-2}\left(b_{11(1)}\right) \alpha^{-1}\left(\phi^{-1}\left(b_{2}\right)_{1}\right)\right) \alpha^{-1}\left(h_{1}\right) \\
& \otimes \beta^{-2}\left(b_{12(0)(0)}\right) \cdot S\left(\alpha^{-2}\left(b_{12(0)(1)}\right)\right) \\
& \otimes\left(\alpha^{-2}\left(b_{12(1)}\right) \alpha^{-1}\left(\phi^{-1}\left(b_{2}\right)_{2}\right)\right) \alpha^{-1}\left(h_{2}\right)
\end{aligned}
$$

$$
\begin{aligned}
& =\beta^{-1}\left(b_{11(0)}\right) \cdot S\left(\alpha^{-2}\left(b_{11(1) 1}\right)\right) \\
& \otimes\left(\alpha^{-3}\left(b_{11(1) 2}\right) \alpha^{-1}\left(\phi^{-1}\left(b_{2}\right)_{1}\right)\right) \alpha^{-1}\left(h_{1}\right) \\
& \otimes \beta^{-1}\left(b_{12(0)}\right) \cdot S\left(\alpha^{-2}\left(b_{12(1) 1}\right)\right) \\
& \otimes\left(\alpha^{-3}\left(b_{12(1) 2}\right) \alpha^{-1}\left(\phi^{-1}\left(b_{2}\right)_{2}\right)\right) \alpha^{-1}\left(h_{2}\right) \\
& =\left(\beta^{-3}\left(b_{1111}\right) \cdot \alpha^{-4}\left(\phi^{-1}\left(b_{1112}\right) \phi\left(b_{112}\right)_{1}\right)\right) \\
& \cdot S\left(\alpha^{-3}\left(\phi\left(b_{112}\right)_{21}\right)\right) \\
& \otimes\left(\alpha^{-4}\left(\phi\left(b_{112}\right)_{22}\right) \alpha^{-1}\left(\phi^{-1}\left(b_{2}\right)_{1}\right)\right) \alpha^{-1}\left(h_{1}\right) \\
& \otimes\left(\beta^{-3}\left(b_{1211}\right) \cdot \alpha^{-4}\left(\phi^{-1}\left(b_{1212}\right) \phi\left(b_{122}\right)_{1}\right)\right) \\
& \cdot S\left(\alpha^{-3}\left(\phi\left(b_{122}\right)_{21}\right)\right) \\
& \otimes\left(\alpha^{-4}\left(\phi\left(b_{122}\right)_{22}\right) \alpha^{-1}\left(\phi^{-1}\left(b_{2}\right)_{2}\right)\right) \alpha^{-1}\left(h_{2}\right) \\
& =\beta^{-2}\left(b_{1111}\right) \cdot \alpha^{-3}\left(\phi^{-1}\left(b_{1112}\right)\right) \\
& \cdot\left(\alpha^{-4}\left(\phi\left(b_{112}\right)_{1}\right) S\left(\alpha^{-5}\left(\phi\left(b_{112}\right)_{21}\right)\right)\right) \\
& \otimes\left(\alpha^{-4}\left(\phi\left(b_{112}\right)_{22}\right) \alpha^{-1}\left(\phi^{-1}\left(b_{2}\right)_{1}\right)\right) \alpha^{-1}\left(h_{1}\right) \\
& \otimes \beta^{-2}\left(b_{1211}\right) \cdot \alpha^{-3}\left(\phi^{-1}\left(b_{1212}\right)\right) \\
& \cdot\left(\alpha^{-4}\left(\phi\left(b_{122}\right)_{1}\right) S\left(\alpha^{-5}\left(\phi\left(b_{122}\right)_{21}\right)\right)\right) \\
& \otimes\left(\alpha^{-4}\left(\phi\left(b_{122}\right)_{22}\right) \alpha^{-1}\left(\phi^{-1}\left(b_{2}\right)_{2}\right)\right) \alpha^{-1}\left(h_{2}\right) \\
& =\beta^{-2}\left(b_{1111}\right) \cdot \alpha^{-2}\left(\phi^{-1}\left(b_{1112}\right)\right) \\
& \left.\otimes \beta^{-2}\left(\phi\left(b_{112}\right)\right) \alpha^{-1}\left(\phi^{-1}\left(b_{2}\right)_{1}\right)\right) \alpha^{-1}\left(h_{1}\right) \cdot \alpha^{-2}\left(\phi^{-1}\left(b_{1212}\right)\right) \\
& \otimes\left(\alpha^{-2}\left(\phi\left(b_{122}\right)\right) \alpha^{-1}\left(\phi^{-1}\left(b_{2}\right)_{2}\right)\right) \alpha^{-1}\left(h_{2}\right) .
\end{aligned}
$$

For all $b \in B, h \in H$, we define $\rho^{\prime}: C \rightarrow H \otimes C$ by

$$
\rho^{\prime}(\bar{b})=\bar{b}_{-1} \otimes \bar{b}_{0}=\phi\left(b_{11}\right) \alpha\left(\phi^{-1}\left(b_{2}\right)\right) \otimes \beta^{-1}\left(\bar{b}_{12}\right),
$$

and $\lambda: C \rightarrow H \otimes H$ is defined by

$$
\begin{aligned}
\lambda(\bar{b})= & \lambda_{1}(\bar{b}) \otimes \lambda_{2}(\bar{b}) \\
= & \phi\left(b_{1}\right) \alpha^{-2}\left(\phi^{-1}\left(b_{22}\right)_{1}\right) \\
& \otimes \alpha^{-1}\left(\phi\left(b_{21}\right)\right) \alpha^{-2}\left(\phi^{-1}\left(b_{22}\right)_{2}\right) .
\end{aligned}
$$

On the other hand, we have

$$
\begin{gathered}
(\bar{\psi} \otimes \bar{\psi}) \Delta_{\lambda}(\bar{b} \otimes h)=(\bar{\psi} \otimes \bar{\psi})\left(\overline { b } _ { 1 } \otimes \left(\alpha^{-4}\left(\bar{b}_{21-1}\right)\right.\right. \\
\left.\cdot \alpha^{-3}\left(\lambda_{1}\left(\bar{b}_{22}\right)\right)\right) \alpha^{-1}\left(h_{1}\right) \otimes \beta^{-2}\left(\bar{b}_{210}\right) \\
\left.\otimes \alpha^{-2}\left(\lambda_{2}\left(\bar{b}_{22}\right)\right) \alpha^{-1}\left(h_{2}\right)\right)=(\bar{\psi} \otimes \bar{\psi})\left(\bar{b}_{1}\right.
\end{gathered}
$$




$$
\begin{aligned}
& \otimes\left(\alpha ^ { - 4 } ( \phi ( b _ { 2 1 1 1 } ) \alpha ( \phi ^ { - 1 } ( b _ { 2 1 2 } ) ) ) \alpha ^ { - 3 } \left(\phi\left(b_{221}\right)\right.\right. \\
& \left.\left.\cdot \alpha^{-2}\left(\phi^{-1}\left(b_{2222}\right)_{1}\right)\right)\right) \alpha^{-1}\left(h_{1}\right) \otimes \beta^{-3}\left(\bar{b}_{2112}\right) \\
& \left.\otimes \alpha^{-2}\left(\alpha^{-1}\left(\phi\left(b_{2221}\right)\right) \alpha^{-2}\left(\phi^{-1}\left(b_{2222}\right)_{2}\right)\right) \alpha^{-1}\left(h_{2}\right)\right) \\
& =(\bar{\psi} \otimes \bar{\psi})\left(\overline { b } _ { 1 } \otimes \left(\alpha^{-3}\left(\phi\left(b_{2111}\right)\right)\right.\right. \\
& \cdot\left(\left(\alpha^{-4}\left(\phi^{-1}\left(b_{212}\right)\right) \alpha^{-4}\left(\phi\left(b_{221}\right)\right)\right)\right. \\
& \left.\left.\left.\cdot \alpha^{-5}\left(\phi^{-1}\left(b_{2222}\right)_{1}\right)\right)\right)\right) \alpha^{-1}\left(h_{1}\right) \otimes \beta^{-3}\left(\bar{b}_{2112}\right) \\
& \otimes \alpha^{-2}\left(\alpha^{-1}\left(\phi\left(b_{2221}\right)\right) \alpha^{-2}\left(\phi^{-1}\left(b_{2222}\right)_{2}\right)\right) \alpha^{-1}\left(h_{2}\right) \\
& =(\bar{\psi} \otimes \bar{\psi})\left(\overline { b } _ { 1 } \otimes \left(\alpha^{-2}\left(\phi\left(b_{211}\right)\right) \varepsilon\left(b_{221}\right)\right.\right. \\
& \left.\left.\cdot \alpha^{-4}\left(\phi^{-1}\left(b_{2222}\right)_{1}\right)\right)\right) \alpha^{-1}\left(h_{1}\right) \otimes \beta^{-2}\left(\bar{b}_{212}\right) \\
& \otimes \alpha^{-2}\left(\alpha^{-1}\left(\phi\left(b_{2221}\right)\right) \alpha^{-2}\left(\phi^{-1}\left(b_{2222}\right)_{2}\right)\right) \alpha^{-1}\left(h_{2}\right) \\
& =(\bar{\psi} \otimes \bar{\psi})\left(\bar{b}_{1} \otimes\left(\alpha^{-2}\left(\phi\left(b_{211}\right)\right) \alpha^{-3}\left(\phi^{-1}\left(b_{222}\right)_{1}\right)\right)\right) \\
& \cdot \alpha^{-1}\left(h_{1}\right) \otimes \beta^{-2}\left(\bar{b}_{212}\right) \otimes \alpha^{-2}\left(\phi\left(b_{221}\right)\right. \\
& \left.\cdot \alpha^{-1}\left(\phi^{-1}\left(b_{222}\right)_{2}\right)\right) \alpha^{-1}\left(h_{2}\right) \stackrel{(83)}{=} b_{11} \cdot \phi^{-1}\left(b_{12}\right) \\
& \otimes\left(\alpha^{-2}\left(\phi\left(b_{211}\right)\right) \alpha^{-3}\left(\phi^{-1}\left(b_{222}\right)_{1}\right)\right) \alpha^{-1}\left(h_{1}\right) \\
& \otimes \beta^{-2}\left(b_{2121} \cdot \phi^{-1}\left(b_{2122}\right)\right) \otimes \alpha^{-2}\left(\phi\left(b_{221}\right)\right. \\
& \left.\cdot \alpha^{-1}\left(\phi^{-1}\left(b_{222}\right)_{2}\right)\right) \alpha^{-1}\left(h_{2}\right)=\beta^{-2}\left(b_{1111}\right) \\
& \cdot \alpha^{-2}\left(\phi^{-1}\left(b_{1112}\right)\right) \otimes\left(\alpha^{-2}\left(\phi\left(b_{112}\right)\right)\right. \\
& \left.\cdot \alpha^{-1}\left(\phi^{-1}\left(b_{2}\right)_{1}\right)\right) \alpha^{-1}\left(h_{1}\right) \otimes \beta^{-2}\left(b_{1211}\right) \\
& \cdot \alpha^{-2}\left(\phi^{-1}\left(b_{1212}\right)\right) \otimes\left(\alpha^{-2}\left(\phi\left(b_{122}\right)\right)\right. \\
& \left.\cdot \alpha^{-1}\left(\phi^{-1}\left(b_{2}\right)_{2}\right)\right) \alpha^{-1}\left(h_{2}\right) \text {. }
\end{aligned}
$$

Therefore, $B \simeq C \rtimes_{\lambda} H \simeq A \square H$ as Hom-coalgebras.

\section{Conflicts of Interest}

The authors declare that there are no conflicts of interest regarding the publication of this paper.

\section{Acknowledgments}

This work was supported by the NNSF of China (no. 11601132), the Natural Science Foundation of Henan Province (no. 152300410086), the Research Fund of PhD of Henan Normal University (no. qd14151), the TianYuan Special Funds of the National Natural Science Foundation of China (no. 11626138), and the NSF of Shandong Province (no. ZR2016AQ03).

\section{References}

[1] A. Makhlouf and S. D. Silvestrov, "Hom-algebra structures," Journal of Generalized Lie Theory and Applications, vol. 2, no. 2, pp. 51-64, 2008.

[2] A. Makhlouf and S. Silvestrov, "Hom-algebras and Homcoalgebras," Journal of Algebra and Its Applications, vol. 9, no. 4, pp. 553-589, 2010.

[3] R. J. Blattner, M. Cohen, and S. Montgomery, "Crossed products and inner actions of Hopf algebras," Transactions of the American Mathematical Society, vol. 298, no. 2, pp. 671-711, 1986.

[4] Y. Doi and M. Takeuchi, "Cleft comodule algebras for a bialgebra," Communications in Algebra, vol. 14, no. 5, pp. 801817, 1986.

[5] R. J. Blattner and S. Montgomery, "Crossed products and Galois extensions of Hopf algebras," Pacific Journal of Mathematics, vol. 137, no. 1, pp. 37-54, 1989.

[6] D. W. Lu and S. H. Wang, "Crossed product Hom-Hopf algebras and lazy 2-cocycle," https://arxiv.org/pdf/1509.01518.pdf.

[7] S. Caenepeel, S. Dascalescu, and S. Raianu, "A Maschke-type theorem for crossed coproclucts," An. Stiint. Univ. Ovidius Constanta Ser. Mat, vol. 2, pp. 44-51, 1994.

[8] S. Dascalescu, G. Militaru, and S. Raianu, "Crossed coproducts and cleft coextensions," Communications in Algebra, vol. 24, no. 4, pp. 1229-1243, 1996.

[9] S. Dascalescu, S. Raianu, and Y. H. Zhang, "Finite HopfGalois coextensions, crossed coproducts, and duality," Journal of Algebra, vol. 178, no. 2, pp. 400-413, 1995.

[10] D. E. Radford, "The structure of Hopf algebras with a projection," Journal of Algebra, vol. 92, no. 2, pp. 322-347, 1985.

[11] S. Caenepeel, B. Ion, G. Militaru, and S. Zhu, "The factorization problem and the smash biproduct of algebras and coalgebras," Algebras and Representation Theory, vol. 3, no. 1, pp. 19-42, 2000.

[12] S. Guo and X.-L. Chen, "A Maschke type theorem for relative Hom-HOPf modules," Czechoslovak Mathematical Journal, vol. 64(139), no. 3, pp. 783-799, 2014.

[13] D. Yau, "Enveloping algebras of Hom-Lie algebras," Journal of Generalized Lie Theory and Applications, vol. 2, no. 2, pp. 95$108,2008$.

[14] D. Yau, "Hom-bialgebras and comodule Hom-algebras," International Electronic Journal of Algebra, vol. 8, pp. 45-64, 2010.

[15] T. Ma, H. Li, and T. Yang, "Cobraided smash product HomHOPf algebras," Colloquium Mathematicum, vol. 134, no. 1, pp. 75-92, 2014.

[16] A. Masuoka and Y. Doi, "Generalization of cleft comodule algebras," Communications in Algebra, vol. 20, no. 12, pp. 37033721, 1992. 


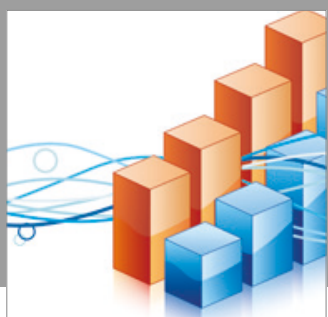

Advances in

Operations Research

vatersals

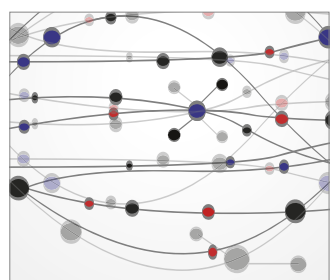

\section{The Scientific} World Journal
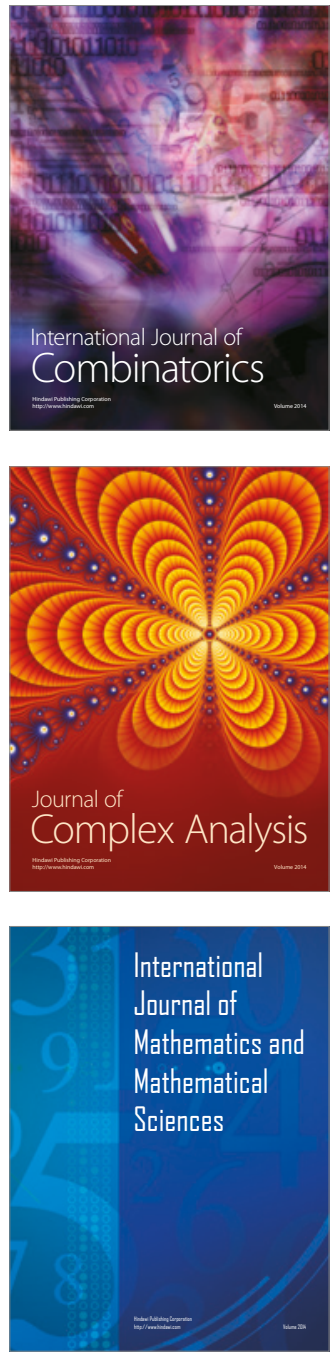
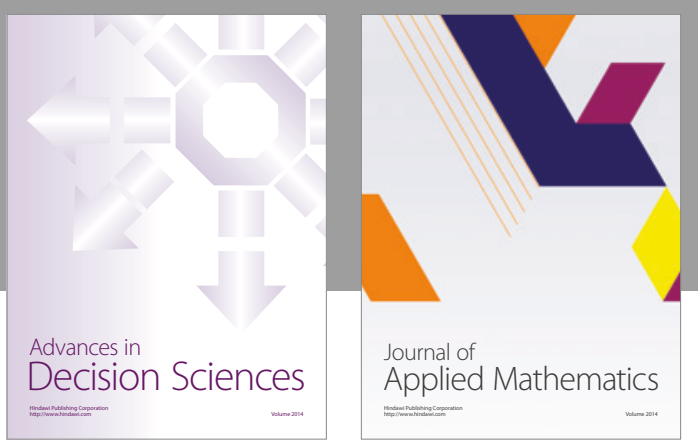

Algebra

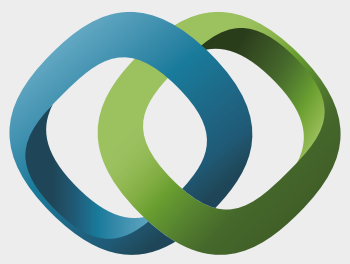

\section{Hindawi}

Submit your manuscripts at

https://www.hindawi.com
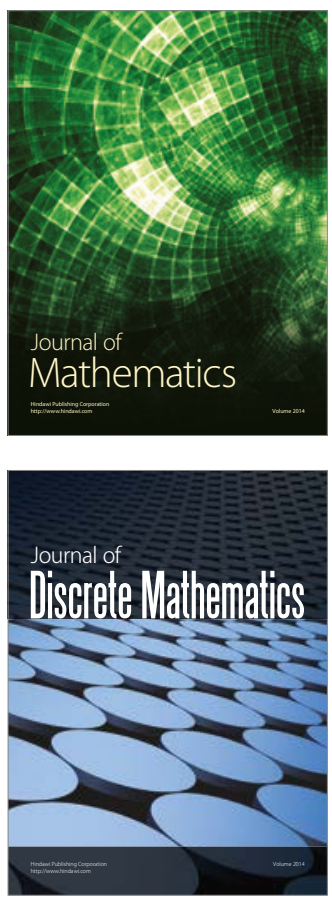

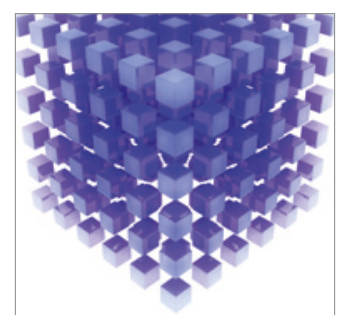

Mathematical Problems in Engineering
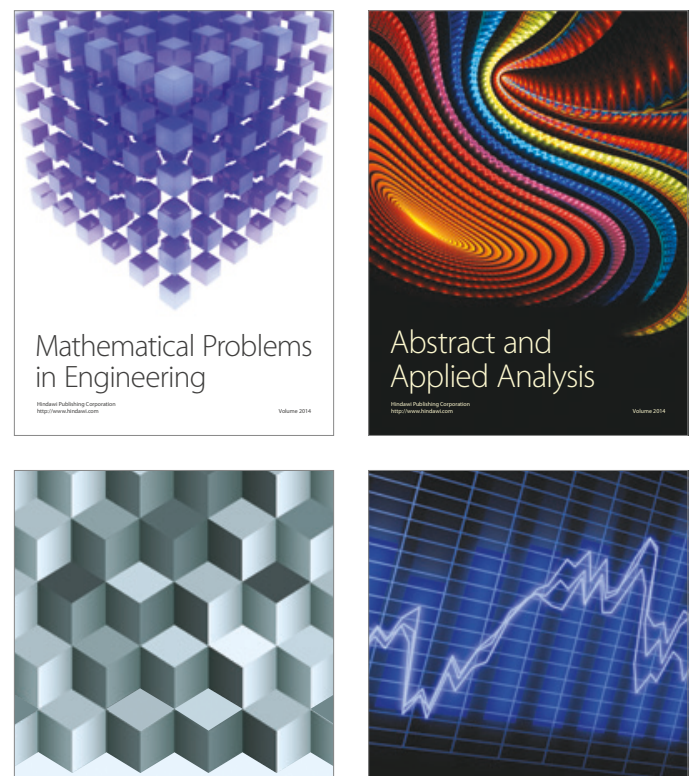

Journal of

Function Spaces

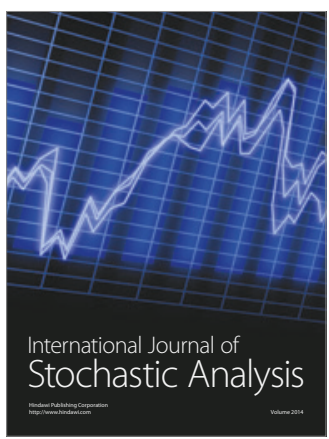

Probability and Statistics
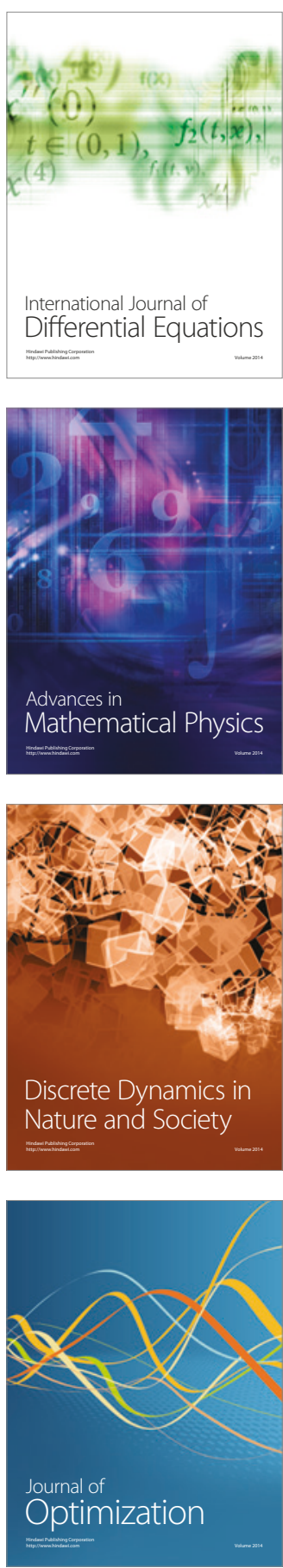